\title{
A review of soft-tissue sarcomas: translation of biological advances into treatment measures
}

This article was published in the following Dove Press journal: Cancer Management and Research

\section{Ngoc T Hoang* \\ Luis A Acevedo* \\ Michael J Mann \\ Bhairavi Tolani}

Thoracic Oncology Program, Department of Surgery, Helen Diller Family Comprehensive Cancer Center, University of California, San Francisco, CA, USA

*These authors contributed equally to this work
Correspondence: Bhairavi Tolani Thoracic Oncology Program, Helen Diller Family Comprehensive Cancer Center, University of California, 1600 Divisadero Street, San Francisco, CA 94II5, USA Email Bhairavi.Tolani@ucsf.edu

\begin{abstract}
Soft-tissue sarcomas are rare malignant tumors arising from connective tissues and have an overall incidence of about five per 100,000 per year. While this diverse family of malignancies comprises over 100 histological subtypes and many molecular aberrations are prevalent within specific sarcomas, very few are therapeutically targeted. Instead of utilizing molecular signatures, first-line sarcoma treatment options are still limited to traditional surgery and chemotherapy, and many of the latter remain largely ineffective and are plagued by disease resistance. Currently, the mechanism of sarcoma oncogenesis remains largely unknown, thus necessitating a better understanding of pathogenesis. Although substantial progress has not occurred with molecularly targeted therapies over the past 30 years, increased knowledge about sarcoma biology could lead to new and more effective treatment strategies to move the field forward. Here, we discuss biological advances in the core molecular determinants in some of the most common soft-tissue sarcomas - liposarcoma, angiosarcoma, leiomyosarcoma, rhabdomyosarcoma, Ewing's sarcoma, and synovial sarcoma - with an emphasis on emerging genomic and molecular pathway targets and immunotherapeutic treatment strategies to combat this confounding disease.
\end{abstract}

Keywords: sarcoma, molecular pathways, immunotherapy, genomics

\section{Introduction}

Soft-tissue sarcoma (STS) is a diverse group of rare cancers that arise from pathological transformations in the mesenchyme, which is the mesodermal portion of the embryo that develops into connective and skeletal tissues. These rare cancers account for $<1 \%$ of all adult malignancies, and an estimated 12,000 new cases of STS are diagnosed in the US each year, with approximately 5,000 deaths. ${ }^{1,2}$ While the exact cause of carcinogenesis has remained elusive and these cancers can arise from any body part, most STSs are diagnosed in the extremities (59.5\%), followed by the trunk $(17.9 \%){ }^{3}$ At the time of initial diagnosis, distant metastases are rarely present, but blood is the most common route for the disease to spread, most frequently to the lungs. ${ }^{4}$

Current treatments for STS often involve multiple modalities, including surgery, radiation, and chemotherapy. There are a few US Food and Drug Administration (FDA)-approved chemotherapeutic drugs for treating STS, such as eribulin, trabectedin, ifosfamide, anthracyclines, and taxanes, but toxicity and partial responses remain significant limitations. Little progress has been made with respect to targeted therapeutics. The 5-year overall survival rate for STS is $90 \%$ for stage I, $81 \%$ for stage II, and 56\% for stage III. ${ }^{5}$ Beyond TNM stage and histologic grade, ${ }^{5,6}$ additional prognostic factors include surgical margins, age, anatomic site, and histologic subtype. ${ }^{7}$ 
According to the World Health Organization classification, over 100 distinct histological subtypes have been categorized. ${ }^{8}$ Given this diversity, the appropriate course of treatment could be guided by a better understanding of the disease pathobiology at the molecular level. In this review, we focus on several of the most common histologic subtypes in adults: liposarcoma (LPS), angiosarcoma, leiomyosarcoma (LMS), rhabdomyosarcoma (RMS), Ewing's sarcoma (ES), and synovial sarcoma (SS). We summarize current knowledge and advances in STS biology in terms of molecular pathways and genomics (Figure 1 and Table 1). We also consider translational implications of new targeted and immunotherapeutic

Liposarcoma

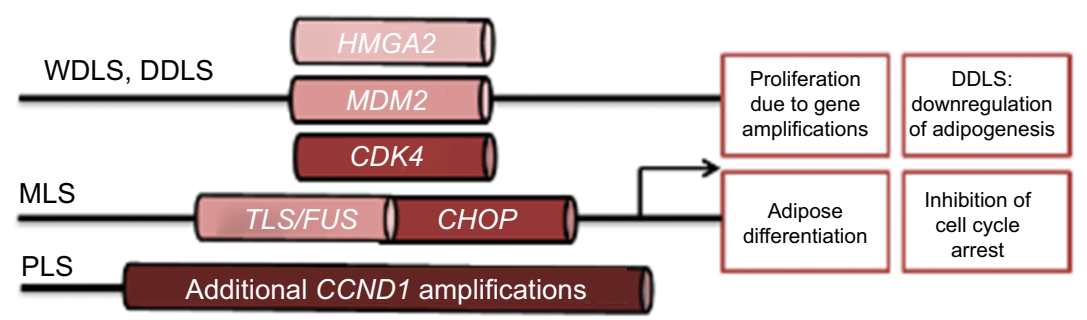

Angiosarcoma

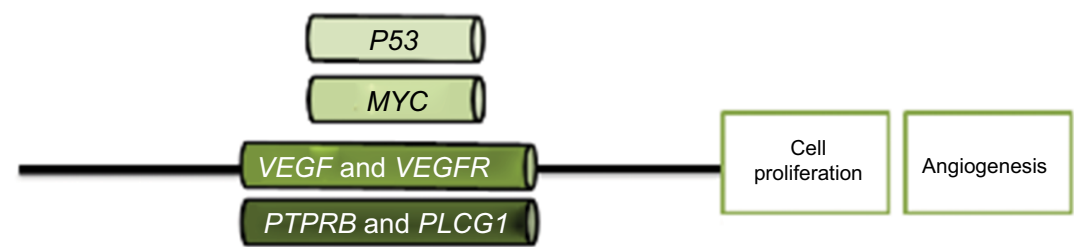

Leiomyosarcoma

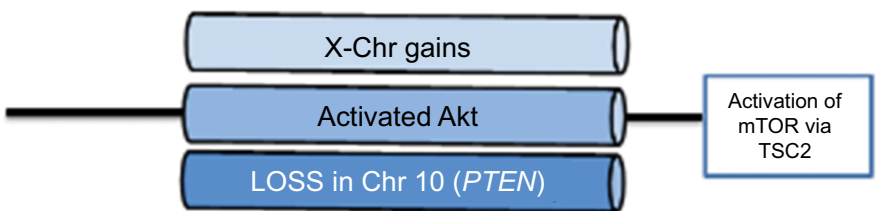

Rhabdomyosarcoma
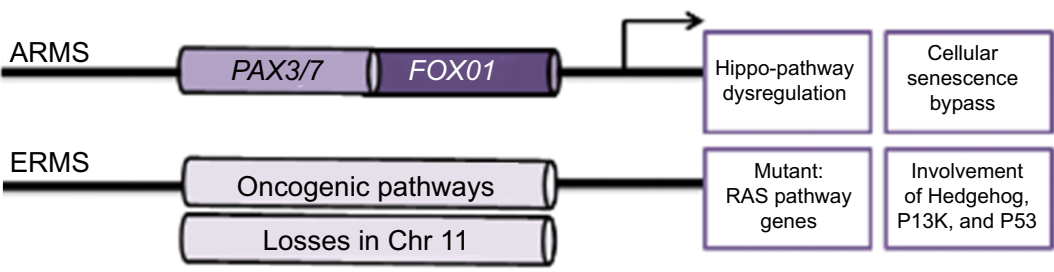

Ewing's sarcoma

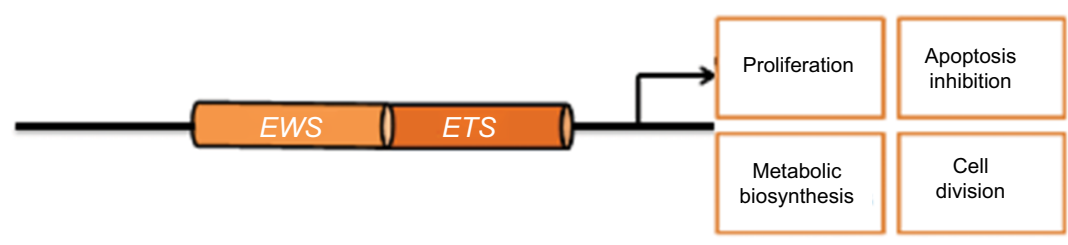

Synovial sarcoma

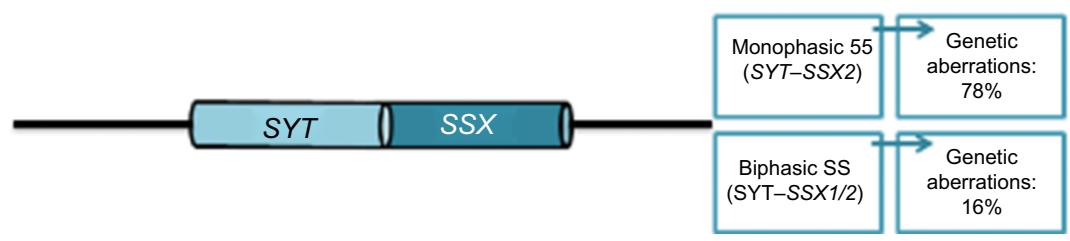

Figure I Genomic changes in soft-tissue sarcoma.

Notes: Liposarcomas consist of four subtypes: well-differentiated liposarcoma (WDLS), dedifferentiated liposarcoma, myxoid liposarcoma (MLS), and pleomorphic liposarcoma (PLS). A common characteristic of WDLS, DDLS, and PLS is amplifications in HMGA2, MDM2, and CDK4. PLS bears additional CCNDI amplifications. MLS, on the other hand, harbors a fusion of TLS/FUS-CHOP, which is responsible for pathogenesis. Angiosarcomas are diverse malignancies and bear aberrations in MYC, VEGF/VEGFR, PTPRB, and PLCGI. Leiomyosarcomas have frequent X-chromosome (Chr) gains, constitutively activated Akt and losses in Chr I0, which bears the PTEN gene. The two latter aberrations lead to mTOR activation via TSC2 and are instrumental in disease pathology. Rhabdomyosarcoma can be subtyped into alveolar rhabdomyosarcoma (ARMS) and embryonic rhabdomyosarcoma (ERMS). The former is associated with PAX3/7-FOXOI fusions and cause Hippo-pathway dysregulation accompanied by bypass of cellular senescence, and the latter is distinguished by losses in Chr II, along with gene mutations in the Ras pathway. Other pathways involved include Hedgehog, PI3K, and p53. Ewing's sarcoma is characterized by EWS-ETS gene fusion, and this potent transcription factor induces genes associated with proliferation, apoptosis inhibition, and metabolic changes to favor biosynthesis and cell division. Synovial sarcoma (SS) is associated with SYT-SSX fusions: SYT-SSX2 for monophasic SS and SYT-SSXI/2 for biphasic SS. Arrows indicate gene transcription. 
Table I Soft-tissue sarcoma genomic landscape

\begin{tabular}{|c|c|c|}
\hline Sarcoma type/sub-type & Gene/chromosome alteration & Frequency \\
\hline \multirow[t]{3}{*}{ LPS (WDLS, DDLS) } & HMGA2 amplification & $76 \%$ \\
\hline & MDM2 amplification & $87 \%$ \\
\hline & CDK4 amplification & $95 \%$ \\
\hline \multirow[t]{4}{*}{ LPS (MLS) } & $13 q 2 \mid-13 q 32$ amplifications & $24 \%$ \\
\hline & Telomerase reactivation & $69 \%$ \\
\hline & Telomerase reactivation & $39 \%$ \\
\hline & C228T TERT mutation & $74 \%$ \\
\hline \multirow[t]{10}{*}{ AS } & TP53 mutation & $4 \%$ \\
\hline & 8q24.2I amplification & $50 \%$ \\
\hline & 10p12.33 amplification & $33 \%$ \\
\hline & 5q35.3 amplification & $11 \%$ \\
\hline & VEGF overexpression & $21 \%-25 \%$ \\
\hline & Inactivating PTPRB mutations & $26 \%$ \\
\hline & Likely activating PLCGI mutations & $9 \%$ \\
\hline & PIK3CA mutations & $3 \%$ \\
\hline & FLT4 mutations & $3 \%$ \\
\hline & H/K/NRAS mutations & $13 \%$ \\
\hline \multirow[t]{3}{*}{ LMS } & Genomic imbalances & $88 \%$ \\
\hline & Aberrant chromosome numbers and structures & $60 \%$ \\
\hline & Promoter hypermethylation of RASSFIA & $39 \%$ \\
\hline \multirow[t]{3}{*}{ LMS (ULMS) } & X-chromosome gains & $48 \%$ \\
\hline & $10 \mathrm{q}$ chromosome region loss & $62 \%$ \\
\hline & I3q chromosome region loss & Most \\
\hline \multirow[t]{3}{*}{ RMS } & Ras pathway mutations & $35 \%-45 \%$ \\
\hline & TP53 mutations & $5 \%-22 \%$ \\
\hline & MDM2 amplification & $10 \%-17 \%$ \\
\hline \multirow[t]{7}{*}{ RMS (ERMS) } & $C D K N 2 A / B$ focal deletion & $23 \%$ \\
\hline & FGFR4 activating mutations & $20 \%$ \\
\hline & NFI locus deletions & $15 \%$ \\
\hline & Ras family activating mutations & $12 \%-42 \%$ \\
\hline & High GLII expression & $21 \%$ \\
\hline & FGFR4 mutations & $9 \%$ \\
\hline & PIK3CA mutations & $5 \%$ \\
\hline \multirow[t]{2}{*}{ RMS (ARMS) } & PAX3-FOXOI gene fusions & $55 \%$ \\
\hline & PAX7-FOXOI gene fusions & $22 \%$ \\
\hline ES & EWS-FLII translocation & Characteristic \\
\hline SS & SYT-SSXI translocation fusion & Characteristic \\
\hline Monophasic SS & Genetic aberrations & $78 \%$ \\
\hline Poorly differentiated SS & Genetic aberrations & $5 \%$ \\
\hline \multirow[t]{3}{*}{ Biphasic SS } & Genetic aberrations & $16 \%$ \\
\hline & Overexpression: KRT5, KRT7, KRT8, KRTI 4 & Preferentially expressed in biphasic samples \\
\hline & Overexpression: EST, ELF3 & Preferentially expressed in biphasic samples \\
\hline
\end{tabular}

Abbreviations: LPS, liposarcoma; WDLS, well-differentiated liposarcoma; DDLS, dedifferentiated liposarcoma; MLS, myxoid liposarcoma; AS, angiosarcoma; LMS, leiomyosarcoma; ULMS, uterine leiomyosarcoma; RMS, rhabdomyosarcoma; ERMS, embryonic rhabdomyosarcoma; ARMS, alveolar rhabdomyosarcoma; ES, Ewing's sarcoma; SS, synovial sarcoma.

strategies under investigative development that could potentially permit longer survival and a better quality of life for those with STS (Table 2).

\section{Liposarcoma}

LPSs are mesenchymal-derived cancers that originate from adipose precursors, named so because of the resemblance they bear to fat cells when examined under microscopy. ${ }^{9}$
These tumors are typically large and bulky, with extensions that branch off from the confines of the main tumor mass. LPS is the most common STS subtype, comprising $20 \%$ of all adult STS. ${ }^{10}$ They most frequently occur in adults over age 40 years, and the 5-year survival rates range from $56 \%$ to $100 \%$ depending upon tumor histology. ${ }^{11}$ Surgery is the standard of care for LPS, but recurrence is common and resistance to chemotherapeutics underscores a critical need 
Table 2 Soft-tissue sarcoma targets, therapeutics, and clinical status

\begin{tabular}{|c|c|c|c|c|}
\hline & Target(s) & Therapeutic & Status & Trial ID \\
\hline LPS & CDK $4 / 6$ & Palbociclib (PD033299I) & Phase II trial completed (20I7) & NCTOI 209598 \\
\hline LPS & VEGFR and PDGFR & Pazopanib & Phase II trial completed (2017) & NCT0I506596 \\
\hline LPS (MLS) & NY-ESOI & CAR T cells & Phase II trial ongoing & NCT02992743 \\
\hline LPS (MLS), SS & Class I MHC expression & Recombinant IFN $\gamma$ & Pilot study ongoing & NCT01957709 \\
\hline LPS (DDLS), LMS & mTOR and CDK $4 / 6$ & Everolimus + ribociclib & Phase II trial ongoing & NCT03।I4527 \\
\hline LPS, LMS & PDLI and DNA repair & Avelumab $(\mathrm{PDLI} \mathrm{mAb})+$ trabectedin & Phase II trial ongoing & NCT030743 \\
\hline LPS, ES, AS (UPS) & PDI and $\mathrm{mTOR}$ & $\begin{array}{l}\text { Nivolumab (PDI mAb) + ABI009 } \\
\text { (mTOR inhibitor) }\end{array}$ & Phase II trial ongoing & NCT0319017 \\
\hline AS & VEGF & Bevacizumab (VEGF mAb) & Phase II trial ongoing & NCT002880I5 \\
\hline LMS, SS & EGFR & Anlotinib (AL38|8) & Phase III trial ongoing & NCT03016819 \\
\hline RMS & $\begin{array}{l}\text { NY-ESOI, MAGEA4, } \\
\text { PRAME, survivin, and SSX }\end{array}$ & TAA-specific CTLs & Phase I trial ongoing & NCT0223986I \\
\hline RMS, ES & Immunomodulated lysis & Recombinant vaccinia GM-CSF (JX594) & Phase I trial completed & NCTOII69584 \\
\hline RMS, SS & CD56 and tubulin & Lorvotuzumab mertansine & Phase II trial ongoing & NCT02452554 \\
\hline ES & EWS-FLII & TK216 & Phase I trial ongoing & NCT02657005 \\
\hline ss & G6PD & DHEA & Phase II trial ongoing & NCT02683I48 \\
\hline ss & mTOR, c-Kit, and PDGFR & Everolimus + imatinib mesylate & Phase II trial completed & NCTOI28I865 \\
\hline SS & $\begin{array}{l}\text { NY-ESOI-expressing } \\
\text { tumor cells }\end{array}$ & $\begin{array}{l}\text { Autologous dendritic cells loaded with } \\
\text { allogeneic tumor lysate expressing } \\
\text { NY-ESOI }\end{array}$ & Phase $1 / I$ ongoing & NCT0I8835I8 \\
\hline $\begin{array}{l}\text { Advanced STS } \\
\text { postchemotherapy }\end{array}$ & $\begin{array}{l}\text { VEGFRI and VEGFR2, } \\
\text { VEGFR3, PDGFR, and } \\
\text { c-Kit }\end{array}$ & Pazopanib & FDA-approved (2012) & - \\
\hline Several & Immunomodulated lysis & HSVI7I6 & Phase I trial ongoing & NCT00931931 \\
\hline Several & Tubulin and mitotic spindle & Eribulin & FDA-approved (2015) & - \\
\hline Several & DNA repair & Trabectedin & FDA-approved (2016) & - \\
\hline Several & PDI and CTLA4 & Nivolumab \pm ipilimumab (CTLA4 mAb) & Phase II trial ongoing & NCT02500797 \\
\hline Several & mTOR & Ridaforolimus & Phase II trial completed (2015) & NCT00II 2372 \\
\hline Several & $\begin{array}{l}\text { VEGFR, PDGFR, and DNA } \\
\text { replication }\end{array}$ & Pazopanib + topotecan & Phase II trial ongoing & NCT023578I0 \\
\hline Several & $\begin{array}{l}\text { Topoisomerase } 2 \text { and } \\
\text { PDGFR }\end{array}$ & Dexrazoxane + doxorubicin + olaratumab & Phase II trial ongoing & NCT025843 \\
\hline Several & $\begin{array}{l}\text { Raf, VEGFRI, VEGFR2, } \\
\text { VEGFR3, PDGFR B, and } \\
\text { c-Kit }\end{array}$ & Sorafenib & Phase II trial completed & NCT00217620 \\
\hline Several & PDLI and NY-ESOI & Atezolizumab (PDLI mAb) + CMB305 & Phase II trial ongoing & NCT026099 \\
\hline Several & $\begin{array}{l}\text { Histone-lysine } \\
\text { methyltransferase EZH2 }\end{array}$ & Tazemetostat & Phase II trial ongoing & NCT0260I950 \\
\hline
\end{tabular}

Abbreviations: LPS, liposarcoma; MLS, myxoid liposarcoma; SS, synovial sarcoma; DDLS, dedifferentiated liposarcoma; LMS, leiomyosarcoma; mAb, monoclonal antibody; ES, Ewing's sarcoma; AS, angiosarcoma; UPS, undifferentiated pleomorphic sarcoma; RMS, rhabdomyosarcoma.

for the identification of novel therapeutic targets. ${ }^{12}$ The most frequent chromosome gains at the genomic level are in chromosome regions $1 \mathrm{q}, 12 \mathrm{q}$, and $13 \mathrm{q} \cdot{ }^{13}$

\section{Liposarcoma: subtypes}

The four recognized histological subtypes of LPS are categorized based on clinicopathological and molecular genetic characteristics: well-differentiated LPS (WDLS), dedifferentiated LPS (DDLS), myxoid LPS (MLS), and pleomorphic LPS (PLS). ${ }^{10,12}$ The most common are WDLS, DDLS, ${ }^{14}$ and MLS. ${ }^{9}$ Those with greatest metastatic potential include DDLS and PLS. ${ }^{12}$ In the following sections, we present recent molecular discoveries in these biological subtypes of LPS.
Well-differentiated and dedifferentiated liposarcoma WDLS and DDLS occur either in the retroperitoneal region or in the extremities. ${ }^{12}$ Patients with retroperitoneal WDLS or DDLS have higher rates of local recurrence and disease-related deaths than those with extremity tumors. ${ }^{12}$ WDLS and DDLS share common underlying genetic alterations, but WDLS in particular consists predominantly of mature adipocytes, along with mixtures of primitive lipoblasts and atypical stromal cells. ${ }^{10,12}$ In contrast, DDLS is believed to be more aggressive and more likely a metastatic progression of WDLS with poorer outcomes. DDLS develops due to the deregulation of normal adipocyte differentiation programs, and is thus characterized by a lack of mature adipocytes. ${ }^{10}$ The exact genetic events 
that prompt this evolution are unclear. ${ }^{12}$ WDLS and DDLS are best treated with surgical resection; ${ }^{12}$ metastatic DDLS is commonly resistant to chemotherapy and radiation. ${ }^{10}$

\section{Myxoid liposarcoma}

MLS and round-cell LPS constitute $30 \%$ of all LPS. ${ }^{14}$ These subtypes frequently occur in the lower extremities, ${ }^{14}$ with metastases observed commonly in the lungs, soft tissue, and bones. ${ }^{15}$ Histologically, these cancers consist of round to oval mesenchymal cells, and MLS is characterized by the presence of lipoblasts, which are adipose precursors at different stages of differentiation, with distinct plexiform capillary patterns and a myxoid or mucous matrix. ${ }^{9}$

\section{Pleomorphic liposarcoma}

PLS comprises $5 \%$ of all LPS, making it the rarest subtype. ${ }^{14}$ Along with DDLS, PLS is also aggressive with metastatic potential and associated with increased disease-related deaths. ${ }^{9}$ These high-grade tumors arise frequently in the retroperitoneum ${ }^{9}$ and the lower extremities, and have a high risk of recurrence. ${ }^{14}$ PLS has unusual histological features ${ }^{9}$ similar to malignant fibrous histiocytoma, but with the presence of adipose differentiation. ${ }^{14,16}$

\section{Liposarcoma: genomic landscape Genomic characteristics of WDLS and progression into DDLS}

A common characteristic of both WDLS and DDLS is the presence of a supernumerary ring (where the two arms of the chromosome are fused together) or a giant marker chromosome (where no structural parts of the chromosome can be identified) with amplifications in the chromosome region 12q13-12q15. ${ }^{13-15,17-19}$ This causes amplification of such genes as $H M G A 2, M D M 2$, and $C D K 4 .{ }^{13-15,17-19}$ The occurrence of both $C D K 4$ and $M D M 2$ amplification is associated with higher local recurrence rates (47\% versus $12.5 \%$ in MDM2-exclusive amplifications). ${ }^{20}$

Although WDLS is locally aggressive, it has relatively little metastatic potential ${ }^{12}$ and exhibits fewer copy number aberrations (CNAs) relative to DDLS: $5.7 \%$ in WDLS versus $21 \%$ in DDLS. ${ }^{10,21}$ Of the 11 chromosomes amplified in WDLSP, the most frequent is $12 q 13-12 q 15$, found in 95\% of cases with $C D K 4$ amplifications, $87 \%$ with $M D M 2$ amplifications, and $76 \%$ with $H M G A 2$ amplifications. ${ }^{10}$ Progression from WDLS to DDLS involves additional genomic alterations ${ }^{10}$ and importantly the downregulation of adipocyte differentiation programs. ${ }^{10}$ Nine CNAs, termed progression-associated CNAs, which are differentially expressed between the two subtypes, could potentially have roles in the progression of WDLS to DDLS. ${ }^{10}$

A major element of dedifferentiation from WDLS to DDLS is the loss or downregulation of adipogenesis. ${ }^{10,21-24}$ Adipocyte-metabolic genes such as $L I P E,{ }^{10,21,23} P L I N,{ }^{23}$ and PLIN2, ${ }^{21}$ among others, are also uniquely absent in DDLS, ${ }^{23}$ thus displaying a distinctive genomic landscape with global suppression of adipogenesis. Expression of genes related to apoptosis ( $B A X, B I R C 5, S U L F 1)$, cytoskeleton arrangement and maintenance (CTNNB1, MARKS, TMP4, PLEC), Rasrelated genes ( $R A B 23, H R A S L S 3, R A B 20)$, transcription factors (TLE4, FOXF2, SOX11), and cell-cycle control (MAPK1, $C D C 2, C C N B 2)$ are differentially expressed between DDLS and WDLS. ${ }^{23}$

\section{Genomic characteristics of MLS}

Interestingly, MLS displays very few genomic imbalances and in particular lacks high amplifications commonly observed in the other subtypes. ${ }^{14,25}$ MLS is characterized by the presence of a unique reciprocal translocation of bands 13q, which encodes for $C H O P$, and p11, which encodes for TLS/FUS on chromosomes 12 and 16 , respectively. ${ }^{9,24,25}$ The resulting translocation, $\mathrm{t}(12 ; 16)(\mathrm{q} 13 ; \mathrm{p} 11)$, forms the fusion protein TLS/FUS-CHOP, which may play a role in adipose differentiation and inhibition of $\mathrm{G}_{1} / \mathrm{S}$ cell-cycle arrest induced by native CHOP proteins. ${ }^{9}$ Amplifications of $13 \mathrm{q}$, specifically 13q21-13q31 and 13q32, are also observed frequently in MLS and are associated with poor overall survival. ${ }^{25}$ Telomerase reactivation is moderate in MLS (39\%), ${ }^{19}$ but the TERT promoter mutation C228T occurs commonly in MLS cases $(74 \%){ }^{26}$

\section{Genomic characteristics of PLS}

PLS is distinguished in having the most chromosome imbalances, ${ }^{14,16,25}$ with more gains and deletions of chromosome regions than any other LPS subtype, occurring on all chromosomes. ${ }^{25}$ Unlike MLS, PLS has not been associated with any translocations; $;^{9,27}$ instead, frequent CNA amplifications occur in a number of chromosome regions. ${ }^{27}$ Specifically, amplification of 13q31-13q32 (frequent in PLS but not other subtypes) is associated with poor patient survival and increased tumor-related death, with a median survival of 35 months versus 78 months in those with no $13 \mathrm{q}$ gain. ${ }^{25}$ PLS displays differentially high amplifications of CCND1 and similarly high amplification of CCND2, MYB, MDM2, GLI1, and $C D K 4$ to DDLS. ${ }^{28}$ 


\section{Chemotherapeutics for LPS: eribulin and trabectedin} In 2015 and 2016, the FDA approved two chemotherapeutic agents specifically for LPS treatment: eribulin and trabectedin. Eribulin acts by inhibiting the polymerization of tubulin, preventing the formation of microtubules, and interfering with the mitotic spindle required for cell division. A Phase II clinical trial showed measurable tumor shrinkage and RECIST (response evaluation criteria in solid tumors) scores in LPS patients treated with eribulin. ${ }^{29}$ About $47 \%$ of patients with DDLS treated with eribulin showed complete or partial response or stable disease. ${ }^{29}$ Approximately $45 \%$ of patients with other LPS subtypes (eg, PLS and MLS) showed stable disease. ${ }^{29}$ In a large Phase III multicenter clinical trial, eribulin treatment significantly extended overall survival in patients by 2 months compared to dacarbazine, a DNA cross-linking agent. ${ }^{30}$ Overall survival was improved in LPS patients treated with eribulin compared to dacarbazine. ${ }^{30}$

Trabectedin exerts its antitumor effect by interfering with DNA repair machinery and by causing DNA breakage and cell-cycle arrest. In 2007, a clinical trial with exclusively MLS patients showed efficacy ( $51 \%$ objective response with progression-free survival at 6 months in $88 \%$ of patients) of the drug in MLS, and specifically for those who carried the type I and II variants of the TLS/FUS-CHOP fusion products. ${ }^{31}$ The efficacy of trabectedin specifically against type I and II variants was confirmed in xenograft models, showing that trabectedin prevented and prolonged the binding of variant types I and II to target genes $P T X 3$ and $N F 1 .{ }^{32}$

\section{Liposarcoma: targeted therapeutics}

Despite the relatively large amount of genomic information garnered for LPS subtypes, there is currently no approved targeted therapy for LPS.

\section{CDK4/6 for WDLS/DDLS}

WDLS and DDLS both harbor $C D K 4$ amplifications, ${ }^{13,14,15,17-19}$ making CDK4 a promising therapeutic target. A Phase II WDLS/DDLS-specific clinical trial showed PD0332991, a CDK4 oral inhibitor, to be effective in the treatment of these subtypes, with $60 \%$ of patients showing no disease progression at 12 weeks and median progression-free survival of 17.9 weeks..$^{33}$ These initial findings of CDK4 inhibition as a potential targeted therapy for LPS are promising, and several clinical trials are ongoing in patients with WDLS/ DDLS. ${ }^{34,35}$ Furthermore, PD0332991, also known as palbociclib, has been approved for the treatment of ER-positive and human EGFR2-negative breast cancer. Given that it is the first CDK inhibitor to be approved as a cancer therapy by the FDA, its use in WDLS/DDLS could become a reality in the near future.

\section{VEGFR/PDGFR inhibition}

Treatment with pazopanib, a small-molecule inhibitor of VEGFR and PDGFR, showed promising results for patients with high-intermediate-grade disease that was surgically unresectable or metastatic. ${ }^{36}$ Overall, $2.4 \%$ of patients had a partial response to the drug, $41.5 \%$ had stable disease, and $43.9 \%$ experienced disease progression; overall and progression-free survival were, respectively, 12.62 and 4.44 months. ${ }^{36}$ A similar trial with pazopanib is ongoing that includes lowgrade subtypes, such as WDLS. ${ }^{37}$ Another clinical trial for all subtypes of LPS is investigating the efficacy of pazopanib in combination with topotecan, a compound that prevents the religation of topoisomerase-dependent DNA-strand breaks during DNA replication. ${ }^{38}$

\section{Akt-mTOR pathway inhibition}

Ridaforolimus, an mTOR inhibitor, influences effector proteins $\mathrm{S} 6 \mathrm{~K}$ and 4E-BP1, and has shown promising results for several sarcomas, including LPS. ${ }^{39}$ In a Phase II clinical trial, ridaforolimus was shown to elicit responses in $30 \%$ of LPS patients; $27 \%$ had progression-free survival at 6 months and median progression-free survival of 14.3 weeks, which is comparable to outcomes from other novel agents, such as trabectedin and pazopanib. ${ }^{39}$ These promising findings led to a Phase III clinical trial. ${ }^{39}$ An ongoing clinical trial is investigating the potential use of another mTOR inhibitor, everolimus, along with a CDK4/6 inhibitor, ribociclib, in patients with advanced DDLS. ${ }^{40}$

\section{Liposarcoma: immunotherapy DDLS and PLS}

Currently, two immunotherapeutic drug candidates are in clinical trials for these two high-grade and aggressive subtypes of LPS. ${ }^{41,42}$ Both trials involve monoclonal antibodies against the immunotargets PDL1 and CTLA4, namely nivolumab and ipilimumab. ${ }^{41,42}$ One trial is investigating the potential neoadjuvant effect of these two drugs in patients with surgically resectable tumors. ${ }^{41}$ The other trial will examine the efficacy of nivolumab with or without ipilimumab in patients with unresectable or metastatic disease. ${ }^{42}$

\section{MLS}

A pilot study of CAR T-cell therapy is ongoing for MLS patients with recurrent or unresectable disease. Treatment consists of administering the patients' own dendritic cells 
genetically engineered to express antigen NY-ESO $1 .{ }^{43}$ Similarly, a Phase II clinical trial is under way looking at the effectiveness of atezolizumab, an anti-PDL1 antibody, combined with CMB305, a dendritic-cell-targeted lentiviral vector containing the NY-ESO1 sequence. ${ }^{44}$ Another ongoing pilot study specific for MLS is investigating whether systemic administration of IFN $\gamma$ can modulate immune-cell infiltration and expression of class II MHC proteins that are expressed on dendritic cells, phagocytes, and antibody-producing B cells. ${ }^{45}$

\section{All liposarcoma subtypes}

Ongoing clinical trials are inclusively testing all LPS subtypes as well, such as an efficacy trial of the combination of trabectedin and avelumab, an anti-PDL1 antibody, in patients with unresectable and/or metastatic disease. ${ }^{46}$ Another study is a Phase I trial of ABI009, an albumin-bound rapamycin compound, along with nivolumab. ${ }^{47} \mathrm{~A}$ third trial is a noninferiority study looking into the effectiveness of combining the standard of care, doxorubicin, with dexrazoxane, a topoisomerase II inhibitor, and olaratumab, an anti-PDGFRA antibody. ${ }^{48}$

\section{Angiosarcoma}

Making up 2\% of all STS, angiosarcomas are malignant tumors that develop in the inner lining of blood vessels and lymphatic tissue. They can be found in almost any part of the body, but occur most frequently in the skin, breast, liver, and spleen. ${ }^{49}$ There are several syndromes (neurofibromatosis, Maffucci syndrome, and Klippel-Trénaunay syndrome) and various exogenous chemicals (vinyl chloride, thorium dioxide, arsenic, radium, and anabolic steroids) that are known risk factors for developing angiosarcoma. ${ }^{49}$ Prognosis is generally poor, since most patients are not diagnosed prior to widespread metastasis. The overall 5-year survival rate is $35 \%,{ }^{49}$ but varies depending on the primary tumor site. Liver and heart angiosarcomas have been found to have as low as no 5-year survival, whereas breast, skin, and soft-tissue angiosarcomas tend to have 5-year survival of 51\%, 43\%, and $74 \%$, respectively. ${ }^{50}$ The current standard of care is surgery and chemotherapy. A better understanding of angiosarcoma genomics could lead to targeted therapies.

\section{Angiosarcoma: subtypes}

Angiosarcomas include a mild form known as epithelioid hemangioendothelioma and more aggressive forms, simply termed angiosarcomas. Epithelioid angiosarcoma is rare and arises in endothelial cells. Angiosarcoma can be divided into five subgroups related to etiology or anatomic location: soft-tissue angiosarcoma (25\%), lymphedema-associated angiosarcoma, radiation-induced angiosarcoma, primary breast angiosarcoma $(8 \%)$, and cutaneous angiosarcoma $(60 \%) .{ }^{49}$ As these names imply, angiosarcoma can arise either as de novo tumors (primary angiosarcoma) or as secondary angiosarcomas due to chronic lymphedema or radiotherapy. ${ }^{49}$ Because of this, women with breast cancer who undergo radiation treatment are at 1,000-fold higher risk of developing secondary angiosarcoma, ${ }^{51}$ making the breast the most common place where radiation and lymphedema-associated sarcomas form. ${ }^{51,52}$ Furthermore, breast angiosarcoma is more aggressive than many other breast cancers and has the tendency to develop rapidly, making it difficult to treat. Although breast angiosarcoma affects deep soft tissue, it does not typically spread to the muscles of the chest wall. Other common primary angiosarcoma tumor sites include the skin and soft tissue, ${ }^{51}$ with the skin of the scalp in elderly patients being the most common site for primary angiosarcoma. ${ }^{49,53}$ Skin angiosarcoma remains refractory to treatment measures and progresses quite rapidly. Soft-tissue angiosarcoma afflicts women and men of all ages equally, and typically presents either as a mass in the affected area or as compression of structures inside the abdomen. Although organ-specific angiosarcomas, such as those of the lungs and heart, share the same fundamental characteristics as other STS, the therapeutic strategy employed is individualized based on the subtype.

\section{Angiosarcoma: genomic landscape}

Angiosarcomas are a heterogeneous group of malignancies harboring a wide range of genetic alterations. Although critical in the pathogenesis of many cancers and even in other sarcoma subtypes like LMS and undifferentiated PLS, the role of TP53 gene alterations in angiosarcoma may be more limited. TP53 mutation and deletion rates have been shown to be as low as $4 \%$ and $0 \%$, respectively, in angiosarcoma, ${ }^{54}$ although other studies indicate higher rates. ${ }^{52}$ Additionally, while mutations reported in the MAPK pathway could serve as potential targets of therapeutic interest, ${ }^{52}$ this review focuses on MYC, VEGF/VEGFR, PTPRB, and PLCG1.

\section{MYC}

$M Y C$ is a proto-oncogene known to play a key role in cellcycle progression and cell proliferation, differentiation, and apoptosis. Mutated or constitutively activated Myc has been implicated in many human cancers. For angiosarcoma in particular, $M Y C$ also plays a key oncogenic role, and the most common alterations are amplifications on chromosome $8 \mathrm{q} 24.21$ (50\%), followed by $10 \mathrm{p} 12.33$ (33\%) and $5 \mathrm{q} 35.3$ 
(11\%). ${ }^{55}$ Furthermore, $M Y C$ gene amplification and protein overexpression in angiosarcoma is well documented ${ }^{52,55-61}$ and is a useful tool in differentiating between primary and secondary angiosarcomas and atypical vascular lesions, which are potential precursors to angiosarcoma. Of the three most common alterations, MYC amplification has been widely shown to occur almost exclusively in secondary angiosarcoma, underscoring the fact that genetic distinction can exist even in morphologically indistinguishable tumors. ${ }^{55,57-60}$ MYC amplification in secondary radiationinduced angiosarcoma can be observed in up to $100 \%$ of samples, as it is an early but often necessary event, ${ }^{58-61}$ whereas atypical vascular lesions have rarer $M Y C$ amplifications ${ }^{58-61}$ Furthermore, $M Y C$ gene amplification is typically, ${ }^{59}$ but not always, related to Myc protein overexpression, suggesting an alternative potential regulatory pathway of MYC expression, such as epigenetic control. ${ }^{56}$ Regardless, the amplification of MYC in many cases of angiosarcoma suggests the importance of its role in the pathogenesis of angiosarcoma and its utility as a diagnostic tool, as well as a potential treatment target utilizing recently described BET inhibitors, among other agents. ${ }^{62}$

\section{VEGFR}

VEGF and its receptor VEGFR play important roles in the angiogenesis of tumor tissue. VEGF overexpression has been shown in $21 \%-25 \%$ of STS patients of various subtypes. ${ }^{63,64}$ Although high VEGF expression correlates significantly with increased tumor grade in various sarcomas, ${ }^{65,66}$ increased VEGF serum levels are related to worse prognoses, particularly in LMS patients, ${ }^{63,64}$ although the use of VEGF as an independent predictor of clinical outcome as a whole remains controversial. ${ }^{66,67}$ Itakura et al examined immunohistochemical staining of VEGF-related proteins in 34 angiosarcoma samples and found positive expression of VEGFA (94\%), VEGFC (12\%), VEGFR1 (94\%), VEGFR2 (65\%), and VEGFR3 (79\%). ${ }^{53}$ Similarly, Antonescu et al showed that in 42 angiosarcoma tumor samples, $60 \%$ expressed VEGFR2 in over $75 \%$ of cells, though no CNAs were detected despite this strong protein expression and clear upregulation at the transcriptional level. ${ }^{68}$ Moreover, Amo et al showed that treating an angiosarcoma cell line (ISO-HAS) with forced expression of VEGF, VEGFR1, and VEGFR2 with recombinant VEGF caused cell growth, further suggesting the importance of the VEGF family in the pathogenesis of angiosarcoma. ${ }^{69}$ Targeting these VEGFrelated proteins in angiosarcoma could thus prove to be an effective treatment.

\section{PTPRB and PLCGI}

Given that aberrant angiogenesis is thought to drive angiosarcoma carcinogenesis, the underlying mutational profile does in fact identify angiogenesis genes PTPRB and PLCG1 in angiosarcoma samples $(\mathrm{n}=39)$ examined via unbiased nextgeneration sequencing. ${ }^{70,71}$ Enrichment of both mutations was highly significant, and Behjati et al showed that 15 of 39 (38\%) tumors had at least one driver mutation in signaling genes involved in angiogenesis. ${ }^{70,71}$ More specifically, 10 of 39 (26\%) samples had inactivating PTPRB mutations, whereas 3 of $34(9 \%)$ samples likely had activating PLCG1 mutations. ${ }^{70}$ PTPRB is a tyrosine phosphatase that inhibits angiogenesis by negatively regulating VGF tyrosine kinases, including VEGFR2, and can often be truncated in angiosarcoma, which may contribute to disease pathogenesis. ${ }^{70} \mathrm{In}$ vitro models have shown that inhibition of PTPRB increases angiogenesis, ${ }^{71}$ so these inactivating PTPRB mutations would be expected to lead to angiogenesis in angiosarcoma, as would the activating PLCG1 mutations. In contrast, $P L C G 1$ encodes for PLC $\gamma 1$, which is a signal transducer of tyrosine kinases. A missense alteration (R707Q) can lead to activation of this enzyme, which has been found in the autoinhibitory cSH2 domain of the protein..$^{70}$ This is consistent with the idea that overactive PLC $\gamma 1$ drives angiosarcoma by constitutive signal transduction downstream of receptor tyrosine kinases, reinforcing PLCG1 as an attractive therapeutic target in angiosarcoma. ${ }^{70}$ In addition to these two genes, other rare mutated genes, such as PIK3CA (of 39 cases), FLT4 ( 1 of 39 cases) and H/K/NRAS (5 of 39 cases) have also been reported in angiosarcoma. ${ }^{70}$

\section{Angiosarcoma: targeted therapeutics Tyrosine kinase inhibitors}

Cytotoxic chemotherapy drugs like ifosfamide, anthracyclines, and taxanes are currently used in treating angiosarcoma. ${ }^{68,72}$ However, recently developed anticancer drugs that target angiogenesis-related proteins are exciting, because of the role of the VEGF family in the pathogenesis of this family of diseases. Overexpressed angiogenesis-related proteins have recently been targeted with tyrosine-kinase inhibitors that inhibit proteins in the VEGF family with the hope that curtailing the blood supply might cause tumor shrinkage. These inhibitors include sunitinib, sorafenib, and pazopanib, which target VEGFR1, VEGFR2, and VEGFR3. ${ }^{68}$

In COS7 cells transfected with two different activating mutant forms of VEGFR2, the tyrosine kinase inhibitors sunitinib and sorafenib were effective in decreasing autophosphorylation of both mutants, suggesting their potential 
for use in angiosarcoma patients bearing mutant VEGFR2. This mutation has been reported in $10 \%$ of samples. ${ }^{68}$ In a recent Phase II study, von Mehren et al showed that sorafenib treatment led to a progression-free rate of $38 \%$ in the vascular sarcoma cohort ( $63 \%$ of which were angiosarcoma patients). ${ }^{73}$ Additionally, the effectiveness of sorafenib in angiosarcoma appears to be related to baseline circulating VEGFA levels, with lower levels being significantly correlated with better outcomes. $^{74}$

The first Phase I dose escalation study of pazopanib in various advanced cancers showed that it was generally well tolerated and had an antitumor activity in a variety of cancers. ${ }^{75}$ Since then, other clinical trials have confirmed both the safety and the efficacy of pazopanib in STS, ${ }^{76}$ establishing it as a viable new treatment option for select STS patients. ${ }^{77}$ Pazopanib proved particularly effective in patients with elevated levels of VEGFR2 in their tumors, as their median overall survival was 7.2 months compared to 2.3 months in those patients with low expression, ${ }^{78}$ further highlighting the value of targeting VEGFR2 overexpression in angiosarcoma as a treatment option.

\section{Anti-VEGF antibody}

Use of the anti-VEGF antibody bevacizumab in angiosarcoma has shown promising results alone and in combination with traditional treatments like surgery, chemotherapy, and radiotherapy. Rosen et al showed that bevacizumab monotherapy in one patient with facial cutaneous angiosarcoma who was unable to undergo traditional treatment showed a well-tolerated and encouraging partial response. ${ }^{79}$ Similarly, a Phase II study of bevacizumab use in angiosarcoma (23 of 30 ) and epithelioid hemangioendothelioma ( 7 of 30) patients reported that $9 \%$ of angiosarcoma patients showed a partial response, and $48 \%$ of angiosarcoma patients had stable disease with an average time to progression of 26 weeks. Bevacizumab was also well tolerated by these patients. ${ }^{80}$ Furthermore, use of bevacizumab in combination with preoperative radiotherapy followed by resection of the tumor bed in two cases of angiosarcoma of the nose was very effective: both patients had a complete response, no residual disease, and no recurrence after follow-up of 8.5 months and 2.1 years. $^{81}$ Fuller et al showed that a combination of bevacizumab with chemotherapy was also effective in even inoperable angiosarcoma, with one patient showing dramatic improvements in appearance and symptoms, which remained stable 11 months after treatment had ended. ${ }^{82}$ Finally, in a Phase II trial of a combination of gemcitabine, docetaxel, and bevacizumab in various STS types, $60 \%$ of angiosarcoma patients showed a partial response to this very exciting group of anticancer drugs. ${ }^{83}$

\section{Angiosarcoma: immunotherapy}

Recently, the advent of immunotherapy has provided promising alternatives to cytotoxic chemotherapeutic agents and targeted therapies in cancer treatment. The most popular immune system targets have been the checkpoint proteins CTLA4 and PD1/PDL1, which can modulate the immune system in the control of cancer progression. Unfortunately, ipilimumab, an anti-CTLA4 antibody, showed no response in six patients with SS. ${ }^{84}$ Immunotargeting of the PD1 receptor and its ligand PDL1 has become increasingly popular in the treatment of multiple cancers, including non-small-cell lung cancer, melanoma, and renal and bladder cancers, and reports of responses to these agents in patients with angiosarcoma have begun to emerge. ${ }^{85}$

\section{Leiomyosarcoma}

LMS tumors that originate from smooth muscle connective tissue account for $10 \%$ of all soft-tissue sarcoma. ${ }^{86} \mathrm{LMS}$ frequently occurs in the extremities, small intestine, or retroperitoneal spaces, or most commonly in the uterus, ${ }^{87}$ hence the categorization into either uterine LMS (ULMS) or nonuterine LMS (NULMS). ${ }^{86,88}$ ULMS, which accounts for $1 \%$ of all uterine malignancies ${ }^{89}$ and $40 \%$ of all uterine sarcomas, ${ }^{86}$ is highly aggressive, with greater metastatic potential than NULMS; ${ }^{86}$ it is also resistant to chemotherapy and radiotherapy. ${ }^{90}$ Median survival in NULMS and ULMS is about 8 and 4.2 years, respectively. ${ }^{86}$ ULMS can progress de novo ${ }^{89}$ or as a result of transformation from uterine leiomyoma, smooth muscle hyperplasia that occurs in as many as $80 \%$ of women. ${ }^{91}$ The documented incidence of transformation from uterine leiomyoma to ULMS, however, is rare $(<0.1 \%) .{ }^{89}$ The 5-year survival rate for LMS is $40 \%$, but decreases to $10 \%-15 \%$ for high-grade $\mathrm{LMS}^{92}$ and $15 \%-25 \%$ for ULMS. ${ }^{90,91}$ The standard of care for LMS is surgical resection when possible. ${ }^{86} \mathrm{LMS}$ can remain dormant for extended periods, and the best outcomes occur after early surgical excision with wide margins. ${ }^{92,93}$ The 5 -year rate of relapse is $40 \%$, which is associated with very high mortality. ${ }^{92,93}$

\section{Leiomyosarcoma: subtypes}

Guo et al confirmed the presence of three molecular subtypes of LMS.${ }^{87}$ Types I and II are linked with extrauterine sites, and type III is closely associated with ULMS. Type I can be identified through immunostaining of overexpressed markers, such 
as ACTG2, SLMAP, LMOD1, CFL2, and MYLK. Type II is characterized by overexpression of $A R L 4 C$, associated with translation, translational elongation, and protein localization, and overexpression of CDK4, CTNNB1, AURKA, RHEB, EGFR, CCND1, MTOR, MAPK1, NOTCH2, and ROR2 has been reported. Type III is associated with upregulation of pathways involved in metabolic processes, ion transport, and regulation of transcription; overexpressed genes include MDM4, ERBB3, EPHA3, ESR1, and EGFR. The identification of these molecular subtypes has been fairly recent, and although more investigations are warranted, these differences could have clinical significance related to the use of existing or novel targeted therapies.

\section{Leiomyosarcoma: genomic landscape}

Genomic imbalances are observed in $88 \%$ of LMS cases, ${ }^{94}$ and $60 \%$ of them have aberrant chromosome numbers and structures. More aberrations are reported in higher grade tumors than in lower grade ones. ${ }^{95}$ LMS tumors have pleomorphic histology, ${ }^{88}$ absence of CD44 variant $3,{ }^{96}$ CD34, c-Kit, and S100 expression, ${ }^{94}$ and complex karyotypes. ${ }^{88}$ Almost half of ULMS cases (48\%) have X-chromosome gains, and the associated amplicons are located near regions containing the androgen receptor, which might potentially contribute to ULMS resistance to hormone therapy. ${ }^{90}$ Other associations include the putative oncogenes $E L K 1$ and $A R A F 1 .{ }^{95}$ Sixty-two percent of ULMS cases display loss of the chromosome region $10 \mathrm{q}$, which harbors the tumor suppressors PTEN and MXI1, and loss of $10 \mathrm{q}$ is associated with recurrent ${ }^{95}$ and higher grade tumors. ${ }^{94,95}$ Most ULMS cases show loss of $13 \mathrm{q}$, the region that houses the tumor suppressor $R B .{ }^{95}$ Loss of $13 \mathrm{q}$, however, is associated with better prognosis than loss of $10 \mathrm{q}$ and contributes to the early development of LMS. ${ }^{95}$ Promoter hypermethylation of the tumor suppressor RASSF $1 A$ occurs in $39 \%$ of LMS, which is higher than in other sarcomas, such as LPS and malignant fibrous histiocytoma, and is associated with poor prognosis in LMS patients with stage II and III cancers. ${ }^{93}$

\section{PTEN-Akt-mTOR pathway in leiomyosarcoma}

Most LMS cases are reported to have activated Akt, ${ }^{88}$ and as mentioned earlier, loss of $10 \mathrm{q}$, which contains the PTEN tumor suppressor gene, is a frequent genomic abnormality found in ULMS and associated with recurrence ${ }^{95}$ and highgrade tumors. ${ }^{94,95}$ Hyperplastic smooth muscle cells that lose PTEN expression then show constitutive activation of $\mathrm{Akt}$, which results in malignant progression into LMS through the release and activation of $\mathrm{mTOR}$ via TSC $2 .{ }^{88}$ Mice deficient in
PTEN in smooth muscle lineage cells have shown decreased life spans, with widespread smooth muscle hyperplasia, mainly in the blood vessels, and urinary and intestinal tracts as early as 1 month. ${ }^{88}$ This was accompanied by rapid onset and an $80 \%$ increase in incidence of LMS as early as 2 months after birth. ${ }^{88}$ Interestingly, there was no appearance of ULMS, suggesting an altered molecular pathogenesis. ${ }^{88}$ When these 1-month-old mice were treated with an mTOR inhibitor, rapamycin, there was a significant increase in life span and a decrease in tumor growth accompanied by decreases in pAkt and mTOR target pS6, emphasizing the crucial role of this pathway in LMS tumorigenesis. ${ }^{88}$

\section{Leiomyosarcoma: targeted therapeutics}

In addition to its use in LPS, the chemotherapeutic agent trabectedin has been approved by the FDA specifically for the treatment of LMS. However, since more than half of early-stage patients experience relapse after therapy, ${ }^{97}$ and the ULMS subtype is resistant to chemotherapy, ${ }^{90}$ more targeted therapies are required for the treatment of LMS.

\section{Aurora kinase $\mathrm{A}$ inhibition}

Proteins that regulate the formation of the mitotic spindle during cell division are frequently overexpressed in a number of cancers. ${ }^{98}$ One of the key players in mitotic spindle organization and stability is aurora kinase A (AurKA), and its expression is highly regulated in ULMS compared with benign LMM or normal myometrial tissue. ${ }^{91}$ Shan et al illustrated the efficacy of an AurKA inhibitor, MK5108, in a mouse model of LMS in which treatment decreased tumor growth and induced $\mathrm{G}_{2} / \mathrm{M}$ cell-cycle arrest and apoptosis. ${ }^{91} \mathrm{~A}$ Phase I clinical trial for the use of MK5108 in solid cancers indicated that it was well tolerated ${ }^{91}$ and provides the impetus for testing this inhibitor in LMS and other sarcomas.

\section{Combination of aurora A kinase and mTOR inhibition}

As mentioned previously, activation of the Akt-mTOR pathway through the loss of tumor suppressor PTEN is crucial for the development of LMS. ${ }^{88,94,95}$ One group is investigating the potential therapeutic benefits of simultaneous AurKA and mTOR inhibition ${ }^{97}$ by using the AurKA inhibitor MLN8237, along with the mTOR inhibitor rapamycin. They found that with a specific schedule of 24 hours of pretreatment with MLN8237 followed by cotreatment for 72 hours with both MLN8237 and rapamycin, tumor volume decreased significantly when compared to MLN8237 or rapamycin alone; this was accompanied by a pronounced decrease in 
cell proliferation and an increase in apoptosis. ${ }^{97}$ Interestingly, LMS subtype II shows overexpression of both MTOR and $A U R K A$, making the dual AurKA and mTOR inhibition regimen a possible personalized therapy for patients with this particular subtype.

\section{ROR2 as potential therapeutic target in leiomyosarcoma}

ROR2 is activated by Wnt5A via the noncanonical Wnt pathway and is highly expressed in several sarcoma subtypes, including LMS. ${ }^{99}$ LMS patients with strong ROR2 staining have worse 5-year disease-specific survival than those with weak or undetectable ROR2 staining. ${ }^{99}$ It is noteworthy that ROR2 expression is consistent between primary and metastatic LMS tumors, which might enable a common treatment for both. ${ }^{99}$ Edris et al showed that ROR2 knockdown led to a $50 \%$ decrease in invasiveness of LMS cell lines and a threefold reduction in average xenograft tumor mass in mice. ${ }^{99}$ ROR 2 expression is specifically enriched in subtype II, making ROR2 another potential therapeutic target for this subtype. Therefore, the use of Wnt pathway inhibitors, such as OTSA101, an anti-Fzd10 antibody in a Phase I trial for advanced SS treatment, might prove to be effective in LMS. ${ }^{100}$

\section{EGFR inhibition}

Using testicular LMS as a model, Sette et al uncovered a putative LMS cancer stem-cell population that was resistant to chemotherapy. ${ }^{101}$ Specifically, this population and differentiated tumor populations have shown high activation of EGFR. ${ }^{101}$ High EGFR expression is also observed in LMS specimens compared to normal tissue. EGFR inhibition combined with chemotherapy results in a decrease in tumor size accompanied by an increase in apoptosis. ${ }^{101}$ As such, EGFR inhibitors could be used to target both cancer stem cells and differentiated tumor populations in LMS. This therapeutic modality can potentially be effective for those with subtype III, who have enriched EGFR expression.

\section{LMP2 as a potential target for ULMS}

$P S M B$ 9/LMP2 encodes a subunit of the proteasome that is involved in antigen processing and frequently genetically altered in ULMS. ${ }^{90}$ LMP2 loss is observed in $85 \%$ of ULMS cases,${ }^{90}$ and over $30 \%$ of samples have essential mutations in $L M P 2 .{ }^{90}$ Female mice with mutated copies of $L M P 2$ spontaneously develop ULMS by 14 months, with $40 \%$ prevalence. ${ }^{102}$ One group showed that inoculation of ULMS cells expressing exogenous LMP2 led to a reduction in tumor growth with no toxicity. ${ }^{90}$ Unfortunately, the proteasome inhibitor bortezomib alone showed the minimal activity in a Phase II study of STS, but one of the patients studied did have a partial response, indicating that combination therapy with other agents may have better effects. ${ }^{103}$ While surgery is a treatment option for resectable ULMS, targeting and reactivation of LMP2 could potentially be efficacious for this category of LMS.

\section{Hepatocyte growth factor/scatter factor}

When hepatocyte growth factor/scatter factor (HGF/SF) is bound to its receptor, c-Met, it promotes angiogenesis, proliferation, and invasion of cancer cells. ${ }^{104}$ Since the c-Met protein is overexpressed in LMS, ${ }^{104}$ it may be a prime target amenable to therapeutic intervention. Burgess et al developed a fully human anti-HGF/SF antibody termed AMG102/ rilotumumab, ${ }^{105}$ which significantly decreased tumor growth when used to treat LMS in tumor-bearing mice. ${ }^{104}$ There have been several completed clinical trials for AMG102 in other nonsarcoma cancers; ${ }^{106-110}$ one, in particular, showed promising results when combined with epirubicin, cisplatin, and capecitabine for gastric and esophagogastric cancers. ${ }^{106}$ Another clinical trial is ongoing for squamous-cell lung carcinoma, ${ }^{111}$ but no study has yet investigated the efficacy of AMG102 in either LMS or other sarcomas. Based upon the encouraging in vivo mouse work and the promising human clinical trials with other types of malignancies, there is strong rationale for testing AMG102 in LMS and other sarcomas.

\section{Leiomyosarcoma: immunotherapy Checkpoint inhibition}

Pembrolizumab, an anti-PDL1 antibody, was approved by the FDA in 2017 for solid tumors. ${ }^{12}$ A number of LMS tumors are positive for PD1 expression, and two clinical trials are investigating the effect of pembrolizumab alone ${ }^{113}$ or in combination with the immunosuppressor cyclophosphamide ${ }^{114}$ in LMS. A different anti-PDL1 antibody, nivolumab, was shown to be an effective treatment for one patient with refractory LMS, who had already undergone surgery and multiple rounds of radiation and chemotherapy. ${ }^{115}$ Furthermore, Paoluzzi et al reported a retrospective study of 24 STS patients treated with nivolumab: seven were diagnosed with LMS, three of whom had stable disease after eight cycles of treatment. ${ }^{112}$ However, the response to pembrolizumab was dramatically different in different individual tumors in a single ULMS patient. ${ }^{116}$ Molecular analysis suggested that loss of PTEN in LMS may correspond to resistance to PD1 inhibition. ${ }^{116}$ Since $P T E N$ loss or its equivalence is a frequent genetic alteration in LMS, ${ }^{88,94,95}$ other immunotherapeutic 
strategies may be considered for patients with these tumors. Overall, immunocheckpoint inhibitors have shown encouraging activity in LMS patients, and studies with expanded cohort enrollment are ongoing to confirm the efficacy of these inhibitors.

\section{CD47 inhibition}

CD47 is a cell surface marker that is overexpressed by cancer cells ( $87 \%$ of cases) relative to normal muscle tissues; CD47 prevents cells from being phagocytized by macrophages of the immune system. ${ }^{92,117}$ LMS has also shown high infiltration of tumor-associated macrophages, which can promote cancer-cell aggressiveness, and patients have shown significantly poorer prognoses ${ }^{92,117}$ Edris et al hypothesized that blocking the antiphagocytosis function of CD47 would allow infiltrated macrophages already present in the LMS tumor to switch from a protumor to antitumor function and eliminate the tumor cells within the tumor. ${ }^{92,117}$ In vitro cellbased studies demonstrated phagocytosis of LMS cells by macrophages when treated with an anti-CD47 antibody. ${ }^{92}$ Anti-CD47 treatment drastically reduced tumor mass in vivo in the range of 5- to 30-fold, with few to no distal metastases compared to the controls; a nearly 70-fold decrease in distal metastases was also reported. ${ }^{92}$ Currently, four different anti-CD47 antibodies are undergoing clinical trials for use in hematological and solid cancers. ${ }^{118-123}$ The aforementioned studies collectively represent an underexploited therapeutic opportunity for treatment of LMS patients with anti-CD47 antibodies.

\section{Rhabdomyosarcoma}

RMSs are highly aggressive tumors that typically develop from skeletal muscle cells. ${ }^{124}$ They represent $3 \%-4 \%$ of all childhood cancers and are the most common childhood and adolescent STS, ${ }^{124,125}$ accounting for $40 \%$ of pediatric STS. ${ }^{126}$ Although RMS can occur anywhere in the body, it most commonly occurs in the head and neck $(10 \%)$, orbit $(9 \%)$, genitourinary tract (24\%), extremities (19\%), and nasal passage and sinuses (16\%). ${ }^{124,127}$ Not only are RMS symptoms tumor site-specific, but prognoses are also linked to primary tumor location. Standard of care depends on primary tumor site and the age of the patient, but can include surgery, radiation, and chemotherapy. RMS frequently metastasizes to the lungs, bone marrow, and bones, and heterogeneity in these tumors makes them confounding and difficult to diagnose, given the lack of strong genetic markers. However, up to $70 \%$ of newly diagnosed cases that do not involve metastases can be cured with multimodal therapy. Since survival rates can vary between $35 \%$ and $90 \%$ depending on the RMS subtype, a clear diagnosis is essential for disease management. ${ }^{128,129}$ Various environmental risk factors have been associated with increased risk of developing RMS, such as paternal smoking, maternal recreational drug use, advanced maternal age, and $\mathrm{X}$-ray exposure in utero. ${ }^{126}$ Additional genetic risk factors include neurofibromatosis type 1, Li-Fraumeni syndrome, Beckwith-Wiedemann syndrome, hereditary retinoblastoma, nevoid basal-cell carcinoma syndrome, Rubinstein-Taybi syndrome, and Costello syndrome. ${ }^{124,126}$ Though advancements in multimodal chemotherapy have shown large increases in patient survival, ${ }^{130,131}$ toxicity remains an issue, and the 5-year survival rate for metastatic disease remains at $30 \%,{ }^{132}$ underscoring the need for additional therapeutic strategies.

\section{Rhabdomyosarcoma: subtypes}

RMS has traditionally been categorized into two main types according to histopathological differences. The most common subtype is embryonic RMS (ERMS), which accounts for about $60 \%$ of RMS, ${ }^{133}$ whereas alveolar RMS (ARMS) accounts for $\sim 20 \%$ of cases. ${ }^{133}$ ERMS usually manifests in the head and neck, genitourinary tract, and retroperitoneum of children $<10$ years of age, whereas ARMS usually occurs in the trunk, arms, and legs of adolescents and young adults. ${ }^{124}$ Clinical outcomes differentiate the two subtypes as well, because outcomes for ERMS are typically considered favorable if the tumor is localized, whereas ARMS has a higher propensity to metastasize and generally has a poorer prognosis. ${ }^{124,132}$ Five-year survival rates for RMS vary depending on the risk group and subtype, ${ }^{130}$ but the overall ERMS 5-year survival rate is $73.4 \%$, whereas the ARMS 5-year survival rate is $47.8 \% .^{126}$

\section{Rhabdomyosarcoma: genomic landscape}

In RMS, the overall somatic mutation burden is relatively low, ${ }^{132}$ but various chromosomal alterations are key and could serve either as prognostic indicators or as targets for therapy.

\section{Alveolar rhabdomyosarcoma}

Eighty percent of ARMS have distinguishing translocations between chromosomes 2 and 13 (t[2;13][q35; q14]) or between chromosomes 1 and $13(\mathrm{t}[1 ; 13][\mathrm{p} 36 ; \mathrm{q} 14])$, which correspond, respectively, to $P A X 3-F O X O 1$ and $P A X 7-$ FOXO1 gene fusions. ${ }^{124,132-134} P A X 3-F O X O 1$ gene fusions have been detected in $55 \%$ of ARMS patients, whereas $P A X 7-F O X O 1$ gene fusions were found in $22 \%$ of ARMS patients. ${ }^{132}$ Although $P A X 3, P A X 7$, and $F O X O 1$ are typically 
transcription factor-encoding genes, the $P A X 3-F O X O 1$ fusion gene produces an even more potent transcription activator than $P A X 3$, suggesting a role in the pathogenesis of ARMS through aberrant upregulation of $P A X 3$ target genes, ${ }^{135,136}$ though $P A X 3-F O X O 1$-specific targets, such as $P D G F R$, have also been shown to be upregulated. ${ }^{137}$ In vitro and in vivo experiments have confirmed that $P A X 3$ and $P A X 3-F O X O 1$ compete for the same targets, and higher $P A X 3-F O X O 1$ embryonic expression leads to impaired neural crest migration and development. ${ }^{136}$ Although $P A X 3-F O X O 1$ expression seemingly plays a critical role in pathogenesis, it alone does not seem to be enough to cause ARMS. Mouse models made to express the gene fusion developed physical abnormalities but no tumors, ${ }^{135,138}$ suggesting the need for coexisting alterations. Potential cooperating events seem to include dysregulation of the Hippo pathway ${ }^{139}$ and $P A X 3-F O X O 1$ bypassing cellular senescence by cooperating with loss of INK4a. ${ }^{140}$ Whole-genome sequencing suggests that the most common cooperating events in $P A X 3 / 7-F O X O 1$ fusions present in RMS are due to genetic amplifications of $M Y C N, C D K 4$, and MIR17-92; deletion of CDKN2A; or loss of heterozygosity of chromosome $11 \mathrm{p} 15.5 .^{124,132}$

Whereas the $P A X 3 / 7$ fusion type was not associated with patient outcome among ARMS patients with localized disease, patients with metastatic ARMS with PAX7-FOXO1 fusion had a 4-year survival rate of $75 \%$ compared to an $8 \%$ 4-year survival rate for those with the $P A X 3-F O X O 1$ gene fusion. ${ }^{134}$ The presence or absence of these $P A X 3 / 7-F O X O 1$ fusions has been used to subcategorize RMS more accurately as a whole, ${ }^{132,141}$ since the prognosis and molecular profiling for fusion-absent ARMS patients are nearly indistinguishable from those of ERMS patients, despite their histological differences. ${ }^{141}$ ARMS samples that were fusion absent also had significantly more somatic mutations than those that were fusion present, ${ }^{132}$ suggesting the need and possibility for alternative treatment strategies between the two. Mutations found in fusion-absent but not fusion-present ARMS include the genes $N R A S$ and $P I K 3 C A$, whereas fusion-positive tumors nearly exclusively showed amplification of the chromosome region $12 q 13-12 q 14,{ }^{132}$ which is associated with worse overall survival. ${ }^{142}$

\section{Embryonic rhabdomyosarcoma}

ERMS most characteristically shows allelic loss at chromosome 11 p.15.5, ${ }^{124}$ a region that appears to include tumorsuppressor genes, and wild-type chromosome 11 transfer into an ERMS cell line causes a decrease in proliferation, ${ }^{143}$ suggesting restoration of tumor suppressor activity.
Studies suggest that in most ERMS, both 11p.15.5 alleles are inactivated, with an inactivated paternal allele being conserved and the maternal allele being lost altogether. ${ }^{124}$ Further chromosomal alterations shown in ERMS include gains of chromosomes 2, 7, 8, 11, 12, 13, 17, 18, and 20; losses of chromosomes $10,14,15$, and $16 ;^{144,145}$ and translocations in the $1 \mathrm{p} 11-1 \mathrm{q} 11$ region. ${ }^{146}$ Moreover, studies have shown that $35 \%$ (5 of 14) of ERMS samples contain mutant $N R A S$ or $K R A S$ genes, ${ }^{147}$ and at least $45 \%$ of fusionabsent RMS as a whole have mutational activations in the Ras pathway, including FGFR4, RAS, NF1, and PIK3CA. ${ }^{132}$ Additional mutations or gene amplifications have also been shown in TP53, MDM2, CDKN2A, GLI1, CTNNB1, and PTPN11. ${ }^{124,132,148-150}$ Though $\mathrm{p} 53$ expression has been shown in ample RMS, ${ }^{151} P 53$ genetic alteration frequencies in RMS seem to vary. Takahashi et al reported $P 53$ gene alterations in $22.2 \%$ (10 of 45 ) of samples, ${ }^{151}$ but Taylor et al reported that only $5 \%$ ( 1 of 20 ) of tumor samples showed P53 mutations. ${ }^{152}$ Despite these differences, neither source reported a correlation between P53 mutation status and prognosis. ${ }^{127,152}$ Both sources also reported similar MDM2 gene amplification frequency in RMS samples, with Takahashi et al reporting amplification in $16.7 \%$ (3 of 18) of samples ${ }^{127}$ and Taylor et al reporting 10\% (2 of 20). ${ }^{152}$ Additionally, Paulson et al showed $C D K N 2 A / B$ focal deletion in 23\% (6 of 26) of ERMS, activating FGFR4 mutations in $20 \%$ (5 of 26) of ERMS, frequent low-level gains of a chromosome region containing GLI1, deletions in the NF1 locus in 15\% (4 of 26) of ERMS, and $R A S$-family activating mutations in $42 \%$ (11 of 26) of ERMS. ${ }^{149}$ Similarly, Pressey et al showed that high expression of GLI1 was present in 21\% (15 of 70) of ERMS tumors, ${ }^{148}$ and Shukla et al showed $R A S$ family mutations in $11.7 \%$ (7 of 60) of ERMS samples, FGFR4 mutations in $9.3 \%$ of ERMS samples, and PIK3CA mutations in $4.9 \%$ of ERMS samples. ${ }^{150}$ Collectively, these studies suggest that p53, RAS, Hedgehog, and PI3K pathways are potentially necessary in the pathogenesis of ERMS and could thus be sensitive to targeted inhibition.

\section{Rhabdomyosarcoma: targeted therapeutics}

Standard chemotherapy treatment from the Soft Tissue Sarcoma Committee of the Children's Oncology Group consists of stratification of patients based on risk groups and undergoing regimens with combinations of vincristine, dactinomycin, cyclophosphamide, and sometimes irinotecan. ${ }^{131}$ Although success has been reported with low-risk and intermediaterisk groups, the 5-year failure-free survival for high-risk 
groups has changed little over the past 25 years. ${ }^{130,133}$ While a greater understanding of the molecular basis of RMS has led to new strategies of stratifying patients into more accurate risk groups to improve chemotherapeutic outcomes, ${ }^{133,153}$ alternative targeted therapies also show promise. One study showed RMS cell lines were sensitive to an IGF1 receptor small-molecule inhibitor. ${ }^{154}$ Similarly, taking advantage of inhibitor pathway activity in RMS, investigators showed in two studies that combination treatment of Ras-MEK-ERK and PI3K-Akt-mTOR pathway inhibitors led to synergistic RMS inhibition in vitro and in vivo. ${ }^{155,156}$ Guenther et al used the dual PI3K-mTOR inhibitor PI103 in combination with the MEK inhibitor U0126 on RMS cell lines and found highly synergistic triggering of apoptotic activity in both histological variants, whereas use of only one drug failed to cause cell death. ${ }^{156}$ Renshaw et al inhibited the same pathways, but used a combination of the TORC1/2 inhibitor AZD8055 and the MEK inhibitor AZD6244, and were able to show synergistic cell growth inhibition in RMS xenografts. ${ }^{155}$ Interestingly, their study also showed a lack of efficacy when just one drug was used, because compensatory cross-talk pathways seemed to render monotherapy ineffective. Finally, Chen et al showed that RMS is susceptible to reactive oxygen species and suggested that therapeutics that increase oxidative stress may synergize with current chemotherapy treatments against RMS. ${ }^{157,158}$

\section{Rhabdomyosarcoma: immunotherapy}

The oncogenic protein Pax3-FoxO1 plays a role in the development of RMS and promotes an immunosuppressive tumor microenvironment ${ }^{153}$ that renders antitumor function by the immune system ineffective. In the following sections are some highlights of recent breakthroughs and current immunorelated clinical trials for RMS.

\section{Selective autologous lymphocyte and immunostimulator regimen}

Although localized RMS is quite treatable, recurrent or metastatic disease is associated with disappointing outcomes. ${ }^{159} \mathrm{In}$ an early clinical trial looking into the efficacy of an adjuvant immunotherapy for recurrent or metastatic RMS, patients received infusions of their own lymphocytes with or without dendritic cells pulsed with the fusion peptide plus IL2, an immunostimulator. ${ }^{159,160}$ The initial results were promising, with $43 \% 5$-year overall survival for those treated with the combination of lymphocytes, peptide-pulsed dendritic cells, and IL2 compared to $31 \%$ among those treated with lymphocytes alone. ${ }^{159} \mathrm{~A}$ second-generation clinical trial, which enrolled similar participants, modified the regimen to induce a greater immunoresponse and antitumor effect. ${ }^{161}$ The new protocol further enriched and purified the lymphocytes to be depleted of $\mathrm{CD} 25^{+}$regulatory $\mathrm{T}$ cells that caused immunosuppression, as well as potential residual tumor cells. The mature dendritic cells were pulsed with the patient's own tumor lysate in place of the fusion peptide, and the immunostimulant added was IL7, as opposed to IL2. ${ }^{159,161}$ The regimen was tolerated well, with no treatment-related high-grade adverse effects. ${ }^{159}$ The 5 -year overall survival rate with this second-generation immunotherapy was $51 \%$, which was significantly higher than the previous regimen, and the progression-free survival was $32 \% .{ }^{159}$ Interestingly, survival on this regimen was higher for RMS patients than for those with other sarcomas, suggesting the specificity of this regimen to those with metastatic or recurrent RMS. ${ }^{159}$

\section{Oncolytic viruses in the treatment of RMS}

Two trials are investigating the effectiveness of two different oncolytic viruses in the treatment of RMS. ${ }^{162,163}$ Oncolytic viruses can specifically target dividing, cancerous cells while sparing the differentiated, normally functioning cells, and both trials are in the early phase of safety and dose escalation testing. One trial, though completed with no results posted, investigated the vaccinia virus armed with an immunostimulatory GM-CSF. ${ }^{162}$ The other trial, ongoing, is examining the antitumor effect of a herpes virus. ${ }^{163}$

\section{Other current clinical trials}

A clinical study of the effect of a tumor lysate vaccine plus the cytokine IL 7 showed very promising initial findings, with $>50 \%$ of participants showing positive immunoresponses. ${ }^{164}$ Another clinical trial that specifically enrolled only RMS patients is testing the feasibility of cytotoxic $\mathrm{T}$ cells armed with tumor-associated antigens and investigating whether an antitumor response can be specifically launched against cancer cells. ${ }^{165}$ The five antigens being tested are SSX, survivin, NY-ESO1, MAGEA4, and PRAME. ${ }^{165}$ Lastly, one clinical trial is investigating the effectiveness of a drug-conjugated antibody, lorvotuzumab mertansine, in which an anti-CD56 antibody is conjugated to the drug mertansine, a tubulin inhibitor, in RMS and other sarcomas. ${ }^{166}$

\section{Ewing's sarcoma}

ES is a neuroectodermal-related malignancy of the bone and soft tissue. ${ }^{167,168}$ ES occurs in children and young adults, ${ }^{169}$ with higher frequency in males. ${ }^{167,168}$ Frequent primary ES sites include the paravertebral region, the chest wall, and the 
lower extremities. ${ }^{167}$ For patients with localized disease, the 5 -year relapse-free survival rate is $50 \%$ for axial primary sites and $67 \%$ for all other sites, but decreases drastically to $21 \%$ for those with detectable metastasis at diagnosis. ${ }^{168}$ However, $30 \%-50 \%$ of those with localized disease will experience relapse within 3 years. ${ }^{167}$ In one study, surgery decreased the relapse rate significantly, from $31 \%$ to $15 \%$ for axial tumors and from $20 \%$ to $4 \%$ for other sites, but patients whose disease relapsed within 2 years typically had worse survival rates. ${ }^{168}$ The hallmark of ES is the translocation fusion between the chromosome regions of the EWS-ETS family of transcription factors. ${ }^{169,170}$ This cancer is grouped together with primitive neuroectodermal tumors and termed the "Ewing family" of tumors due to the presence of similar EWS-ETS translocations. The difference between the two is a continuum of neural differentiation, with one end of the spectrum being primitive neuroectodermal tumor with its differentiated neural phenotype predominantly found in soft tissue, and the other being ES with its undifferentiated neural components. ${ }^{167}$ The extent of differentiation is not a significant prognostic factor for patients with Ewing family tumors. ${ }^{129,171}$

\section{ES: subtypes}

ES tumors are tightly compacted; comprise small, rounded malignant cells separated by strands of fibrous tissue; and contain little to no intercellular stroma. ${ }^{169} \mathrm{ES}$ tumors arise from embryonic osteochondrogenic progenitors that positively express ERG, GDF5, and PTHLH. ${ }^{172} \mathrm{ES}$ and the Ewing family of tumors are characterized by the translocation of EWS and the ETS family of transcription factors, mainly in FLII ( $\geq 85 \%$ of cases) and ERG (10\% of cases), ${ }^{167,170,173}$ and even some rarer fusions with $E T V 1, E 1 A F$, and $F E V^{169}$ $(1 \%-5 \%) .{ }^{173}$ There are four structural variants of $E W S-E R G$ fusion transcripts and up to 18 possible variations of $E W S$ FLI1 transcripts, with the most common being types I and II. ${ }^{167}$ Previously, type I showed significantly higher median overall survival compared to other transcript types ( 9 versus 2 years), ${ }^{167}$ but with recent treatment advancements for ES this disparity is now equalized. ${ }^{174}$ There are very few mutated genes observed in ES related to signaling pathways and chromatin-modifying genes. ${ }^{175}$ Infrequently but consistently, aberrations are detected in three genes: STAG2 (15\%-17\%), CDKN2A (12\%-22\%), and TP53 (6\%-7\%). ${ }^{175,176}$ STAG2 and $C D K N 2 A$ mutations are mutually exclusive and observed in primary tumors, as well as cell lines. ${ }^{176}$ Patients carrying STAG2 or TP53 mutations or both have much lower survival rates. ${ }^{176}$ Interestingly, while only $6 \%$ of ES patients show aberrations in TP53, this increases to $25 \%$ after treatment, suggesting the role of TP53 deregulation in treatment resistance and recurrence. ${ }^{175}$

\section{ES: genomic landscape}

The translocation fusion between EWS and ETS family members produces a potent oncogenic transcription factor $^{177}$ capable of inducing tumorigenesis through increased cell viability and proliferation, ${ }^{178-181}$ apoptosis inhibition, ${ }^{180}$ metabolic changes to favor biosynthesis, and subsequent cell division. ${ }^{182}$ EWS-ETS regulates cell proliferation and anchorage-independent growth in ES cells, but not in a nonES cell line. ${ }^{178}$ It also regulates cell viability through $L R W D 1$, which plays a role in stabilizing the origin recognition complex required for precise DNA replication. ${ }^{181}$ Additionally, EWS-ETS induces autophagy in ES through overexpression of an autophagy-related gene, $A T G 4 B$, which leads to a higher rate of proliferation and lower rate of apoptosis. ${ }^{180}$ Metabolism in $\mathrm{ES}$ is also altered due to EWS-ETS oncogenic regulation, which increases serine biosynthesis via PGHDH upregulation, for the production of proteins, lipids, and nucleic acids to meet the demands of cell proliferation. ${ }^{182}$ Interestingly, elevated PGHDH expression is highest in ES compared to other cancer cell lines, as well as normal tissue, and patients who are deemed at high risk show upregulation in PGHDH. ${ }^{182}$ Inhibition of PGHDH decreases cell proliferation in ES, but not in other non-ES cell lines, suggesting this PGHDHdependent metabolic phenotype is found exclusively in ES. ${ }^{182}$

Metastasis is a crucial factor leading to mortality in ES. ${ }^{168}$ Recently, Choo et al demonstrated the importance of TWIST1, a transcription factor involved in early development, in ES metastasis. ${ }^{183}$ TWIST1 silencing in an in vivo xenograft model showed decreased metastatic burden, and regardless of metastasis status in patients, positive expression of TWIST1 showed a trend toward lower survival. ${ }^{183}$ Another recent discovery was the oncogenic potential of $K D M 3 A$, a histone demethylase, in ES. ${ }^{184} K D M 3 A$ silencing in vitro showed a $50 \%$ decrease in migration and invasion and a tenfold decrease in metastatic burden compared to controls (in mice). ${ }^{184} M C A M$, which is also involved in metastasis in other cancers, is a direct downstream target of $K D M 3 A$, and when silenced, recapitulated impaired proliferation and metastasis are observed with $K D M 3 A$ silencing. $M C A M$ was also significantly associated with poor survival in patients. ${ }^{184}$ ES cells with elevated expression of APLP2, a prosurvival mediator, are resistant to irradiation as well as immune-cell killing via lymphokine-activated killer cells, ${ }^{185}$ allowing cells to continue to grow after treatment and metastasize. 


\section{ES: targeted therapies}

Without systemic chemotherapy, most ES patients develop rapid tumor recurrence. ${ }^{186}$ Standard chemotherapeutic treatment includes combinations of vincristine, actinomycin D, cyclophosphamide, doxorubicin, etoposide, and ifosfamide. ${ }^{186,187}$ Although the 5-year survival rate of patients with localized disease is relatively high (70\%), thanks to recent advancements in diagnosis, surgery, chemotherapy, and radiation, survival rates for patients with metastatic or recurrent disease remain $<25 \% .{ }^{186}$ Long-term toxic effects of treatment continue to be a major issue, ${ }^{188,189}$ further emphasizing the need for new forms of therapy.

Because of the characteristic presence of the fusion gene $E W S-F L I 1$ in ES, it has been the target of various therapeutic attempts that have yielded promising in vitro and in vivo results. Use of a small molecule, YK4-279, to bind to EWSFLII, thereby inhibiting its usual binding and transcriptional modulation, caused apoptosis induction in ES cells and reduced the growth of ES orthotopic xenografts. ${ }^{190} \mathrm{~A}$ recent study found synergism in the inhibition of EWS-FLII activity between YK4-279 use in combination with vincristine both in vitro and in vivo. ${ }^{191}$ The YK4-279 analog TK216 is currently being used in a Phase I clinical trial in patients with relapsed or refractory ES. ${ }^{192}$

Moreover, although trabectedin is a chemotherapeutic, trabectedin sensitivity has been shown to be specifically associated with changes in EWS-FLII transcription factor activity, because drug treatment decreased the expression of several downstream targets of the fusion gene. ${ }^{193}$ Despite this, a Phase II clinical trial using trabectedin in patients with recurrent RMS, ES, and non-RMS soft-tissue sarcomas showed the insufficient activity of the drug as monotherapy. ${ }^{194}$ By taking advantage of downstream targets, Grohar et al were able to show that trabectedin led to the inhibition of the EWSFLI1-downstream Werner syndrome protein, which in turn made ES cell lines hypersensitive to the chemotherapeutic SN38, the active metabolite of irinotecan. ${ }^{195}$ Utilizing this combination of trabectedin and subsequent SN38 treatment, Grohar et al were able to cause regression of two ES xenografts with low drug concentrations and minimal toxicity. ${ }^{195}$ A clinical trial with this combination has indeed begun, but results have not yet been posted. ${ }^{196}$

\section{IGFR I and combination treatments}

Insulin-dependent signal transduction plays an important role in the malignancy of ES. Prieur et al demonstrated that the fusion protein EWS-FLI1 can directly bind to the promoter and represses the expression of a regulator of the
IGF-signaling pathway, IGFBP3, which is a tumor suppressor that disrupts interaction between the receptor IGFR1 and its ligand IGF, is crucial to prosurvival pathways. ${ }^{197}$ Several anti-IGF1R-inhibiting antibodies have been investigated in clinical trials, and in addition to showing a $10 \%$ response rate in ES patients, they were generally well tolerated. ${ }^{198,199}$ Resistance to IGF1R inhibition, however, can occur, ${ }^{200}$ and according to Lamhamedi-Cherradi et al, cells resistant to dalotuzumab, an IGFR1-inhibiting antibody, upregulated some mTOR pathway components. The combination of IGF1R and mTOR inhibition can synergistically bypass the resistance developed from single treatment of either agent and induce an antitumor response. ${ }^{200} \mathrm{~A}$ clinical trial investigating this combination of IGFR1 and mTOR inhibition showed that $29 \%$ of ES patients had tumor reduction and two patients' tumors regressed. ${ }^{201}$ One patient, who had been previously treated with another IGF1R antibody but developed resistance, had a complete response with this combination treatment. ${ }^{201}$

\section{PARP inhibition}

Another potential therapeutic target for treating ES is PARP, a chromatin-associated enzyme involved in DNA repair. Due to upregulation of PARP, ES has proved to be very sensitive to the PARP inhibitor olaparib. ${ }^{202}$ Heske et al recently showed that combined inhibition of PARP and NAMPT, an enzyme crucial for the production of $\mathrm{NAD}^{+}$, a PARP substrate, resulted in delayed tumor growth and increased survival. ${ }^{203}$ A Phase II clinical trial for PARP inhibition in refractory ES showed the treatment was safe and well tolerated, though there was no significant response, potentially because the small cohort consisted of patents who had previously been treated with chemotherapy ${ }^{204}$ Several ongoing clinical trials are examining the efficacy of PARP inhibition specifically in patients with defective DNA damage repair pathways ${ }^{205}$ or in combination with other chemotherapeutic drugs. ${ }^{206}$

\section{ES: immunotherapy}

Breakthroughs in immunobased treatments, notably with regard to PDL1/PD1-inhibiting antibodies, have led to successful treatments in several cancers. Recently, the FDA approved a fourth PDL1-specific antibody - durvalumab. Following are some of the major advances in immunotherapy for the treatment of ES.

\section{Immunoblockage: PDLI status and efficacy}

Recently, Spurny et al discovered a lack of PDL1 expression in ES primary samples as well as established cell lines, though 
surprisingly over half the samples showed positive staining for the receptor-PD1. ${ }^{12}$ Raj et al showed that $30 \%$ of tumors from ES patients expressed PDL1, and PDL1 expression was associated with treatment response. ${ }^{207}$ Le et al recently showed that responsiveness to PDL1 inhibition was due to the lack of a mismatch repair mechanism regardless of tumor type,${ }^{208}$ raising the possibility of the mismatch repair pathway being implicated in the efficacy of this treatment in ES. A Phase II clinical trial has been set to investigate the efficacy of PDL1 inhibition in a variety of cancers, including ES with mismatch repair status as the selection criteria. ${ }^{209}$

\section{$\mathrm{CHMI}$ and $\mathrm{EZH} 2$}

CHM1, an endochondral bone protein, was reported by von Heyking et al to be highly expressed in $\mathrm{ES}^{210,211}$ and is implicated in stemness, enhanced proliferation, invasiveness, and metastasis. ${ }^{210} \mathrm{CHM} 1$ recognition by $\mathrm{T}$ cells can launch an immunoresponse against CHM1-expressing ES cells and significantly inhibit lung and liver metastatic burdens in vivo, suggesting the clinical potential of CHM1 as a target in ES treatment. ${ }^{212}$ Another potential target is EZH2, a histone methyltransferase, shown by Thiel et al to induce an immunoresponse and ES-specific cytotoxicity when primed with allorestricted T cells. ${ }^{211}$

\section{Vaccine-based immunotherapy}

A pilot study investigating the effect of the Vigil/FANG vaccine showed promising results. ${ }^{213}$ Vigil/FANG consists of autologous tumor cells transfected with recombinant GM-CSF and short-hairpin RNA against furin. ${ }^{213}$ When administered to the tumor site, GM-CSF can induce immunoresponses, whereas furin silencing can block activation of immune tolerance activity of TGF $\beta_{1} / \mathrm{TGF} \beta_{2}$, ultimately resulting in tumor destruction. ${ }^{213}$ Most of the participants had a complete knockdown of TGF $\beta_{1} / \mathrm{TGF} \beta_{2}$, all had systemic tumor-specific immunoresponses, and it was estimated that $75 \%$ would survive past 1 year. ${ }^{213}$ The vaccine was well tolerated, and this led to a Phase II clinical trial of the vaccine combined with two chemotherapeutic drugs: temozolomide and irinotecan. ${ }^{214}$

Another ongoing clinical trial is looking into the efficacy of a vaccine containing CD25-depleted lymphocytes with tumor lysates and primed dendritic cells with or without IL7 in patients with high-risk ES. ${ }^{215}$ Initial findings showed 57\% of those vaccinated with immunostimulatory IL 7 had a positive immunoresponse, and $40 \%$ of the patients were reported to be stable or without progressive disease. ${ }^{215}$

\section{Synovial sarcoma}

SS gets its name from its microscopic similarity and proximity to the synovium, which is a specialized connective tissue that lines synovial joints, but in reality the development of tumor cells is not necessarily of synovial origin. While it is an STS typically found in the arms or legs and usually close to tendon sheaths and joint capsules, it can also occur in other locations, such as the heart, brain, and prostate. SS accounts for $\sim 5 \%-10 \%$ of all STS ${ }^{216}$ and $10 \%-20 \%$ of STS in adolescents and young adults; ${ }^{217,218}$ the median age of diagnosis is 35 years, though ages can range 5-85 years. ${ }^{217,219}$ Current standard of treatment includes surgery and radiotherapy, with SS displaying some sensitivity to chemotherapeutic agents like anthracyclines and ifosfamide. ${ }^{217}$ Overall outcomes are poorer in adults, with the 5-year cancer-specific survival rate being $83 \%$ for children and adolescents, but only $62 \%$ in adults; even among adolescents and children, younger patients have better outcomes. ${ }^{220}$ The extremities are the most common site of tumor origin, accounting for $\sim 70 \%$ of cases $;^{220} \sim 50 \%$ of patients exhibit metastatic disease, with $74 \%-81 \%$ of those experiencing metastasis to the lungs. ${ }^{216,220}$ Smaller tumors and those restricted to the extremities portend better outcomes. ${ }^{220}$ Despite differences in survival outcomes among varying ages, histological features of SS between children and adults seem to be identical. ${ }^{220}$

\section{Synovial sarcoma: subtypes}

Although its cellular origin is unclear (its name is counterintuitive, because SS actually may not be of synovial origin), ${ }^{216,221} \mathrm{SS}$ is generally divided into three histological subtypes: monophasic, biphasic, and poorly differentiated. ${ }^{216,220,221}$ Monophasic SS is characterized by the presence of spindle cells and the absence or near-absence of glandular epithelial cells, whereas biphasic SS has equal presence of both spindle cells and glandular epithelial cells. ${ }^{216,221}$ In addition, monophasic SS displays fibrous and sarcomatous cells that are relatively uniform and small and form sheets. In contrast, biphasic SS presents with an epithelial appearance. Furthermore, poorly differentiated SS shows similarities to the small round cells found in ES. ${ }^{216,221}$ Another characteristic of $\mathrm{SS}$ is the unique chromosomal translocation ( $\mathrm{t}[\mathrm{X} ; 18])$, which results in fusion of the $S Y T$ gene to the $S S X 1, S S X 2$, or, on rare occasions, the $S S X 4$ gene..$^{217,220,222}$ A cytogenetic approach that makes use of reverse-transcription polymerase chain reactions can help to differentiate the monophasic and biphasic forms. 


\section{Synovial sarcoma: genomic landscape}

Initially, SS was categorized in the miscellaneous soft-tissue tumor group by the World Health Organization, due to its unknown origin, despite the misleading name, as it bears no resemblance to synovial cells. ${ }^{223,224}$ The discovery of the translocation fusion $S Y T-S S X$ is a now a key characteristic of SS, with SYT-SSX1 found primarily in biphasic SS, but in rare cases also in monophasic SS, whereas SYT-SSX2 is found only in the monophasic subtype. ${ }^{223,225-228}$ Those patients with the biphasic subtype appear to have longer survival than those with the monophasic counterpart, but more data are needed to corroborate these findings. ${ }^{229}$ Similarly, those with SYT-SSX1 have better survival outcomes compared to those with the SYT-SSX2 fusion product. ${ }^{229}$ Overall, 55\% of all SS cases showed changes in the DNA CN and chromosome arms. ${ }^{230}$ There is a higher frequency of genetic aberrations in monophasic subtypes ( $78 \%$ of cases) compared to only $16 \%$ and $5 \%$ of biphasic and poorly differentiated SS, respectively. ${ }^{230} E R B B 2$ and IGFBP2 are genes highly expressed in the epithelial region of the biphasic subtype, potentially indicating the involvement of these genes in epithelial differentiation programs in the biphasic subtypes. ${ }^{231}$ Additionally, E-cadherin and $\alpha$-catenin are preserved in the epithelial components of the biphasic subtype and are correlated with longer survival rates. ${ }^{232}$ Expression of E-cadherin is also associated with low mitotic rates. ${ }^{232}$

The most frequent gains occur on chromosomes 2, 8, and $12,{ }^{230}$ and in particular comprise $M D M 2, M S H 2, K C N K 12$, DCC, CDK2, ERBB3, SAS, and CDK4. ${ }^{229}$ Conversely, the most frequent losses occur on chromosomes 3 and $13,{ }^{230}$ with common losses of HRAS, RASSF1, and CCND1. ${ }^{229}$ Over 50\% of SS cases show positive expression for EGFR, over $40 \%$ express SALL2, and the majority show the presence of $\mathrm{Bcl} 2$ (91\%), pancytokeratin (77\%), EMA (75\%), and cytokeratin 7. ${ }^{233}$ Using a cDNA microarray, Nagayama et al discovered that SS clustered together with another STS type: malignant peripheral nerve sheath tumors (MPNSTs). ${ }^{223}$ However, Terry et al later showed that TLE staining can differentiate SS from MPNST: $>90 \%$ of SS samples expressed TLE, but $<5 \%$ of MPNSTs stained positively for TLE. ${ }^{234}$ Nagayama et al also discovered some commonly upregulated genes in SS to be crucial for neural crest development, migration, and differentiation, suggesting the potential origin of SS was derived from neural crest cells.223

\section{Synovial sarcoma: targeted therapeutics}

Several targeted therapies are currently being tested in clinical trials. ${ }^{235-238}$ One trial that is currently recruiting patients is examining the efficacy of inhibiting glucose-6-phosphate dehydrogenase, which is crucial for tumorigenesis of SS. ${ }^{235}$ Two clinical trials, one for pediatric patients and the other for adults, are investigating the effectiveness of inhibiting a polycomb group protein - EZH2. ${ }^{236,237}$ Another trial is investigating the effect of inhibiting the angiogenic receptors VEGFR2 and VEGFR3. ${ }^{238}$ Several clinical trials are also examining combination regimens. ${ }^{239,240}$ One investigated the combination of an mTOR inhibitor, everolimus, and a multiple TKR inhibitor (c-Kit and PDGFR) - imatinib. The findings were quite promising: of nine participants analyzed, five showed stable disease and four had progression, for a response rate of $55 \% .{ }^{239} \mathrm{~A}$ trial of dacarbazine, a chemotherapeutic agent, together with sorafenib, an Raf/VEGFR2/PDGFR $\beta$ inhibitor, resulted in a response rate of $>70 \%$ : 5 patients had partial responses, 22 had stable disease, and 8 had disease progression, with one death due to adverse effects. ${ }^{240}$

\section{Synovial sarcoma: immunotherapy}

The use of the SYT-SSX fusion product as a target for immunotherapy in SS has shown some therapeutic benefit. ${ }^{241-243}$ Suminoe et al showed that vaccination of dendritic cells armed with the SYT-SSX peptide was well tolerated, with no adverse effects in one patient whose disease had been stable for 2 months. ${ }^{242}$ Similarly, though using only the fusion peptide, Kawaguchi et al also demonstrated a patient's tolerance to the vaccine. The patient had no disease progression, along with a decrease in circulating cytotoxic $\mathrm{T}$ lymphocytes, suggesting these were potentially localized at the site of the tumor, though no biopsy was performed. ${ }^{241}$ Kawaguchi et al further demonstrated the efficacy of the fusion peptide vaccine in conjunction with the administration of an immunostimulant - IFN $\alpha .{ }^{243}$ Half the patient cohort had stable disease, as opposed to only $11 \%$ of patients in the vaccine-only arm. ${ }^{243}$ One caveat of these clinical trials is small samples, owing to the rarity of this type of sarcoma, making it difficult to see the full potential in utilizing the fusion peptide as a therapeutic target.

NY-ESO1 is a tumor antigen belonging to the testis family that is highly expressed in malignant tissues and in the testis, but not in other normal tissues. ${ }^{244,245}$ Over half of SS patients express NY-ESO $1,{ }^{244}$ and because it is immunogenic, ${ }^{246-248}$ it is an attractive target for immunotherapy. In a first-ever clinical trial looking into the effect of administering NYESO1 receptor-expressing autologous $\mathrm{T}$ cells in patients with metastatic SS, Robbins et al reported a response rate of $>60 \%{ }^{245}$ Four of six patients showed partial responses, with the longest being 18 months, and several individuals 
also had regressed lung metastases. ${ }^{245}$ The treatment was well tolerated, leading to the initiation of other similar clinical trials looking into the effectiveness of NY-ESO1 in immunotherapy. ${ }^{249-251}$ One currently active clinical trial is examining the efficacy of combining atezolizumab, a PDL1 inhibitor, with a dendritic cell-specific vector containing the gene for NY-ESO1. ${ }^{252}$ Another clinical trial took a different approach in that allogeneic tumor lysate expression using NY-ESO1 was used to prime patients' autologous dendritic cells. ${ }^{253}$ Upon completion, and depending upon the results, these investigational new therapies could be used in the clinic as treatment measures for SS patients.

\section{Epigenetic changes in STS clinical samples}

Epigenetic regulators are more recently identified therapeutic targets for STS, and small-molecule inhibitors that inhibit them could prove to be useful in treating the disease. In particular, such regulators as DNA methyltransferases, histone deacetylases (HDACs), and the histone-modifying enzyme EZH2 have been implicated in the pathogenesis of several types of STS. ${ }^{254}$ Kawaguchi et al ${ }^{255}$ used clinical samples to demonstrate that the promoter methylation status of tumorrelated genes could have an association with the pathogenesis of STS. Currently, however, no DNA methylation inhibitors have been tested for use in STS.

Histone modifications like phosphorylation, acetylation, methylation, and ubiquitination are crucial in the regulation of genes, including oncogenes and tumor suppressors. In particular, acetylation changes modulated by HDAC occur in several malignancies, including STS. Preclinical treatment with PCI24781, an HDAC inhibitor, has been shown to inhibit growth and cause apoptosis in several STS cell lines, including SS and uterine sarcoma. ${ }^{254}$ Also, EZH2 is an important histone methyltransferase and, when overexpressed in STS samples, is correlated with greater tumor size, metastases, and poor progression. ${ }^{256}$ In particular, EZH2 mediates the expression of the tumor suppressor ERG1 via SYT-SSX in SS. ${ }^{256}$ Also, elevated levels of EZH2 are found in RMS and ES. ${ }^{257}$ As such, EZH2 inhibition might prove successful as a therapeutic approach for STS. Currently, the EZH2 inhibitor tazemetostat is being tested in a Phase II multicenter clinical trial for patients with SS. ${ }^{257}$

\section{Conclusion}

Given the genetic and histological diversity of this large family of cancers, the management of adult STS calls for a multidisciplinary approach to achieve optimal outcomes.
Over the past 30 years, our knowledge of STS biology has progressed very little when compared with other malignancies. This trend has slowly started to change, and additional developments could lead to new therapeutic strategies and treatment options. Several clinical investigations of therapies outlined in this review for STS are under way, and these summarized in Table 2.

\section{Disclosure}

The authors report no conflicts of interest in this work.

\section{References}

1. Jemal A, Tiwari RC, Murray T, et al. Cancer statistics, 2004. CA Cancer J Clin. 2004;54:8-29.

2. Siegel RL, Miller KD, Jemal A. Cancer statistics. CA Cancer J Clin. 2015;65:5-29.

3. Lawrence W Jr, Donegan WL, Ntarajan N, Mettlin C, Beart R, Winchester D. Adult soft tissue sarcomas: a pattern of care survey of the American College of Surgeons. Ann Surg. 1987;205:349-359.

4. Christie-Large M, James SL, Tiessen L, Davies AM, Grimer RJ. Imaging strategy for detecting lung metastases at presentation in patients with soft tissue sarcomas. Eur J Cancer. 2008;44:1841-1845.

5. American Cancer Society. Survival by stage of soft tissue sarcoma. 2017. Available from: https://www.cancer.org/cancer/soft-tissuesarcoma/detection-diagnosis-staging/survival-rates.html. Accessed March 28, 2018.

6. Edge SB, Byrd DR, Compton CC, Fritz AG, Greene FL, Trotti A. Cancer Staging Manual. 7th ed. Heidelberg: Springer; 2010.

7. Zagars GK, Ballo MT, Pisters PW, et al. Prognostic factors for patients with localized soft-tissue sarcoma treated with conservation surgery and radiation therapy: an analysis of 1225 patients. Cancer. 2003;97:2530-2543.

8. Fletcher CD, Bridge JA, Hogendoorn PC, Mertens F. WHO Classification of Tumours of Soft Tissue and Bone. 4th ed. Geneva: World Health Organization; 2013.

9. Kuroda M, Ishida T, Horiuchi H, et al. Chimeric TLS/FUS-CHOP gene expression and the heterogeneity of its junction in human myxoid and round cell liposarcoma. Am J Pathol. 1995;147:1221-1227.

10. Crago A, Socci ND, DeCarolis P, et al. Copy number losses define subgroups of dedifferentiated liposarcoma with poor prognosis and genomic instability. Clin Cancer Res. 2002;18:1334-1340.

11. Bell T. What is liposarcoma? 2012. Available from: http://sarcomahelp. org/liposarcoma.html. Accessed March 28, 2018.

12. Crago AM, Singer S. Clinical and molecular approaches to welldifferentiated and dedifferentiated liposarcoma. Curr Opin Oncol. 2011;23:373-378.

13. Kanojia D, Nagata Y, Garg M, et al. Genomic landscape of liposarcoma. Oncotarget. 2015;6:42429-42444.

14. Rieker RJ, Weitz, Lehner B, et al. Genomic profiling reveals subsets of dedifferentiated liposarcoma to follow separate molecular pathways. Virchows Arch. 2010;456:277-285.

15. Antonescu CR, Tschernyavsky SJ, Decuseara R, et al. Prognostic impact of P53 status, TLS-CHOP fusion transcript structure, and histological grade in myxoid liposarcoma: a molecular and clinicopathologic study of 82 cases. Clin Cancer Res. 2001;7:3977-3987.

16. Rieker RJ, Joos S, Bartsch C, et al. Distinct chromosomal imbalances in pleomorphic and in high-grade dedifferentiated liposarcomas. Int J Cancer. 2002;99:68-73.

17. Spinella-Jaegle S, Rawadi G, Kawai S, et al. Sonic hedgehog increases the commitment of pluripotent mesenchymal cells into osteoblastic lineage and abolishes adipocytic differentiation. $J$ Cell Sci. 2001;114:2085-2094. 
18. Gobble RM, Qin LX, Brill ER, et al. Expression profiling of liposarcoma yields a multigene predictor of patient outcome and identifies genes that contribute to liposarcomagenesis. Cancer Res. 2011;71:2697-2705.

19. Costa A, Daidone MG, Daprai L, et al. Telomere maintenance mechanisms in liposarcomas: association with histologic subtypes and disease progression. Cancer Res. 2006;66:8918-8924.

20. Italiano A, Bianchini L, Gjernes E, et al. Clinical and biological significance of CDK4 amplification in well-differentiated and dedifferentiated liposarcomas. Clin Cancer Res. 2009;15:5696-5703.

21. Tap WD, Eilber FC, Ginther C, et al. Evaluation of well-differentiated/ de-differentiated liposarcomas by high-resolution oligonucleotide array-based comparative genomic hybridization. Genes Chromosomes Cancer. 2011;50:95-112.

22. Taylor BS, DeCarolis PL, Angeles CV, et al. Frequent alterations and epigenetic silencing of differentiation pathway genes in structurally rearranged liposarcomas. Cancer Discov. 2011;1:587-597.

23. Singer S, Socci ND, Ambrosini G, et al. Gene expression profiling of liposarcoma identifies distinct biological types/subtypes and potential therapeutic targets in well-differentiated and dedifferentiated liposarcoma. Cancer Res. 2007;67:6626-6636.

24. Coindre JM, Pédeutour F, Aurias A. Well-differentiated and dedifferentiated liposarcomas. Virchows Arch. 2010;456:167-179.

25. Schmidt H, Bartel F, Kappler M, et al. Gains of 13q are correlated with a poor prognosis in liposarcoma. Mod Pathol. 2005;18:638-644.

26. Koelsche C, Renner M, Hartmann W, et al. TERT promoter hotspot mutations are recurrent in myxoid liposarcomas but rare in other soft tissue sarcoma entities. J Exp Clin Cancer Res. 2014;33:33.

27. Sreekantaiah C. The cytogenetic and molecular characterization of benign and malignant soft tissue tumors. Cytogenet Cell Genet. 1998;82:13-29.

28. Fritz B, Schubert F, Wrobel G, et al. Microarray-based copy number and expression profiling in dedifferentiated and pleomorphic liposarcoma. Cancer Res. 2002;62:2993-2998.

29. Schoffski P, Ray-Coquard IL, Cioffi A, et al. Activity of eribulin mesylate in patients with soft-tissue sarcoma: a phase 2 study in four independent histological subtypes. Lancet. 2011;12:1045-1052.

30. Schoffski P, Chawla S, Maki RG, et al. Eribulin versus dacarbazine in previously treated patients with advanced liposarcoma or leiomyosarcoma: a randomised, open-label, multicenter, phase 3 trial. Lancet. 2016;387:1629-1637.

31. Grosso F, Jones RL, Demetri GD, et al. Efficacy of trabectedin in advanced pretreated myxoid liposarcomas: a retrospective study. Lancet Oncol. 2007;8:595-602.

32. Giandomenico SD, Frapolli R, Bello E, et al. Mode of action of trabectedin in myxoid liposarcoma. Oncogene. 2014;33:5201-5210.

33. Dickson MA, Tap WD, Keohan ML, et al. Phase II trial of the CDK4 inhibitor PD0332991 in patients with advanced CDK4-amplified welldifferentiated or dedifferentiated liposarcoma. J Clin Oncol. 2013;31: 2024-2028.

34. Memorial Sloan Kettering Cancer Center. Study of abemaciclib in dedifferentiated liposarcoma. Available from: https://clinicaltrials.gov/ ct2/show/NCT02846987. NLM identifier: NCT02846987. Accessed March 28, 2018.

35. Hadassah Medical Organization. A phase II single arm study assessing efficacy and safety of ribociclib in patients with advanced well-differentiated or dedifferentiated liposarcoma. Available from: https://clinicaltrials.gov/ct2/show/NCT02571829. NLM identifier: NCT02571829. Accessed March 28, 2018.

36. Vector Oncology. A phase II study of pazopanib in the treatment of surgically unresectable or metastatic liposarcoma. Available from: https://clinicaltrials.gov/ct2/show/NCT01506596. NLM identifier: NCT01506596. Accessed March 28, 2018.

37. Grupo Espanol de Investigacion en Sarcomas. Phase II clinical trial of pazopanib to evaluate the activity and tolerability in patients with advanced and/or metastatic liposarcoma who have relapsed following standard therapies or for whom no standard therapy exists. Available from: https://clinicaltrials.gov/ct2/show/NCT01692496. NLM identifier: NCT01692496. Accessed March 28, 2018.
38. Northwestern University. A phase II study of pazopanib with oral topotecan in patients with metastatic and non-resectable soft tissue and bone sarcomas. Available from: https://clinicaltrials.gov/ct2/show/ NCT02357810. NLM identifier: NCT02357810. Accessed March 28, 2018.

39. Chawla SP, Staddon AP, Baker LH, et al. Phase II study of the mammalian target of rapamycin inhibitor ridaforolimus in patients with advanced bone and soft tissue sarcomas. J Clin Oncol. 2012;30:78-84.

40. MD Anderson Cancer Center. Study of neoadjuvant checkpoint blockade in patients with surgically resectable undifferentiated pleomorphic sarcoma and dedifferentiated liposarcoma. Available from: https://clinicaltrials.gov/ct2/show/NCT03307616. NLM identifier: NCT03307616. Accessed March 28, 2018.

41. National Cancer Institute. Randomized phase II study of nivolumab with or without ipilimumab in patients with metastatic or unresectable sarcoma. Available from: https://clinicaltrials.gov/ct2/show/ NCT02500797. NLM identifier: NCT02500797. Accessed March 28, 2018.

42. Fox Chase Cancer Center. Phase II trial of ribociclib and everolimus in advanced dedifferentiated liposarcoma (DDL) and leiomyosarcoma (LMS). Available from: https://clinicaltrials.gov/ct2/show/ NCT03114527. NLM identifier: NCT03114527. Accessed March 28, 2018.

43. Adaptimmune. A pilot study of NY-ESO- $1^{2259} \mathrm{~T}$ cells in subjects with advanced myxoid/round cell liposarcoma. Available from: https://clinicaltrials.gov/ct2/show/NCT02992743. NLM identifier: NCT02992743. Accessed March 28, 2018.

44. Fred Hutchinson Cancer Research Center. Avelumab and trabectedin in treating patients with liposarcoma or leiomyosarcoma that is metastatic or cannot be removed. Available from: https://clinicaltrials.gov/ ct2/show/NCT03074318. NLM identifier: NCT03074318. Accessed March 28, 2018.

45. Immune Design. A randomized, open-label, phase 2 trial of CMB305 (sequentially administered LV305 and G305) and atezolizumab in patients with locally advanced, relapsed, or metastatic sarcoma expressing NY-ESO-1. Available from: https://clinicaltrials.gov/ct2/show/ NCT02609984. NLM identifier: NCT02609984. Accessed March 28, 2018.

46. Sarcoma Oncology Research Center. A phase 1B single center investigation of safety/efficacy of nivolumab (Opdivo) and ABI-009 (nab-rapamycin) in patients with advanced undifferentiated pleomorphic sarcoma, liposarcoma, chondrosarcoma, osteosarcoma and Ewing sarcoma. Available from: https://clinicaltrials.gov/ct2/show/ NCT03190174. NLM identifier: NCT03190174. Accessed March 28, 2018.

47. Washington University School of Medicine. A non-inferiority study of doxorubicin with upfront dexrazoxane plus olaratumab for the treatment of advanced or metastatic soft tissue sarcoma. Available from: https://clinicaltrials.gov/ct2/show/NCT02584309. NLM identifier: NCT02584309. Accessed March 28, 2018.

48. Lamm W, Natter C, Schur S, et al. Distinctive outcome in patients with non-uterine and uterine leiomyosarcoma. BMC Cancer. 2014;14:981.

49. Young RJ, Brown NJ, Reed MW, Hughes D, Woll PJ. Angiosarcoma. Lancet Oncol. 2010;11:983-991.

50. Fayette J, Martin E, Piperno-Neumann S, et al. Angiosarcomas, a heterogeneous group of sarcomas with specific behavior depending on primary site: a retrospective study of 161 cases. Ann Oncol. 2007;18: 2030-2036.

51. Strobbe LJ, Peterse HL, van Tinteren H, Wijnmaalen A, Rutgers EJ. Angiosarcoma of the breast after conservation therapy for invasive cancer, the incidence and outcome: an unforseen [sic] sequela. Breast Cancer Res Treat. 1998;47:101-109.

52. Murali R, Chandramohan R, Möller I, et al. Targeted massively parallel sequencing of angiosarcomas reveals frequent activation of the mitogen activated protein kinase pathway. Oncotarget. 2015;6:36041-36052.

53. Itakura E, Yamamoto H, Oda Y, Tsuneyoshi M. Detection and characterization of vascular endothelial growth factors and their receptors in a series of angiosarcomas. $J$ Surg Oncol. 2008;97:74-81. 
54. Italiano A, Chen CL, Thomas $\mathrm{R}$, et al. Alterations of the p53 and PIK3CA/AKT/mTOR pathways in angiosarcomas: a pattern distinct from other sarcomas with complex genomics. Cancer. 2012;118:5878-5887.

55. Manner J, Radlwimmer B, Hohenberger P, et al. MYC high level gene amplification is a distinctive feature of angiosarcomas after irradiation or chronic lymphedema. Am J Pathol. 2010;176:34-39.

56. Shon W, Sukov WR, Jenkins SM, Folpe AL. MYC amplification and overexpression in primary cutaneous angiosarcoma: a fluorescence in-situ hybridization and immunohistochemical study. Mod Pathol. 2014;27:509-515.

57. Laé M, Lebel A, Hamel-Viard F, et al. Can c-Myc amplification reliably discriminate postradiation from primary angiosarcoma of the breast? Cancer Radiother. 2015;19:168-174.

58. Fernandez AP, Sun Y, Tubbs RR, Goldblum JR, Billings SD. FISH for MYC amplification and anti-MYC immunohistochemistry: useful diagnostic tools in the assessment of secondary angiosarcoma and atypical vascular proliferations. J Cutan Pathol. 2012;39:234-242.

59. Ginter PS, Mosquera JM, MacDonald TY, D'Alfonso TM, Rubin MA, Shin SJ. Diagnostic utility of MYC amplification and anti-MYC immunohistochemistry in atypical vascular lesions, primary or radiationinduced mammary angiosarcomas, and primary angiosarcomas of other sites. Hum Pathol. 2014;45(4):709-716.

60. Guo T, Zhang L, Chang NE, Singer S, Maki RG, Antonescu CR. Consistent MYC and FLT4 gene amplification in radiation-induced angiosarcoma but not in other radiation-associated atypical vascular lesions. Genes Chromosomes Cancer. 2011;50:25-33.

61. Mentzel T, Schildhaus HU, Palmedo G, Büttner R, Kutzner H. Postradiation cutaneous angiosarcoma after treatment of breast carcinoma is characterized by MYC amplification in contrast to atypical vascular lesions after radiotherapy and control cases: clinicopathological, immunohistochemical and molecular analysis of 66 cases. Mod Pathol. 2012;25:75-85.

62. Tolani B, Gopalakrishnan R, Punj V, et al. Targeting Myc in KSHVassociated primary effusion lymphoma with BET bromodomain inhibitors. Oncogene. 2014;29;33:2928-2937.

63. Potti A, Ganti AK, Tendulkar K, et al. Determination of vascular endothelial growth factor (VEGF) overexpression in soft tissue sarcomas and the role of overexpression in leiomyosarcoma. J Cancer Res Clin Oncol. 2004;130:52-56.

64. Potti A, Ganti AK, Foster H, et al. Immunohistochemical detection of HER-2/neu, c-Kit (CD117) and vascular endothelial growth factor (VEGF) overexpression in soft tissue sarcomas. Anticancer Res. 2004;24:333-337.

65. Pakos EE, Goussia AC, Tsekeris PG, Papachristou DJ, Stefanou D, Agnantis NJ. Expression of vascular endothelial growth factor and its receptor, KDR/Flk-1, in soft tissue sarcomas. Anticancer Res. 2005;25:3591-3596

66. Chao C, Al-Saleem T, Brooks JJ, Rogatko A, Kraybill WG, Eisenberg B. Vascular endothelial growth factor and soft tissue sarcomas: tumor expression correlates with grade. Ann Surg Oncol.2001;8:260-267.

67. Ranieri G, Mammì M, Di Paola EE, et al. Pazopanib a tyrosine kinase inhibitor with strong anti-angiogenetic activity: a new treatment for metastatic soft tissue sarcoma. Crit Rev Oncol Hematol. 2014;89:322-329.

68. Antonescu CR, Yoshida A, Guo T, et al. KDR activating mutations in human angiosarcomas are sensitive to specific kinase inhibitors. Cancer Res. 2009;69:7175-7179.

69. Amo Y, Masuzawa M, Hamada Y, Katsuoka K. Expression of vascular endothelial growth factor in a human hemangiosarcoma cell line (ISOHAS). Arch Dermatol Res. 2001;293:296-301.

70. Behjati S, Tarpey PS, Sheldon H, et al. Recurrent PTPRB and PLCG1 mutations in angiosarcoma. Nat Genet. 2014;46:376-379.

71. Mellberg S, Dimberg A, Bahram F, et al. Transcriptional profiling reveals a critical role for tyrosine phosphatase VE-PTP in regulation of VEGFR2 activity and endothelial cell morphogenesis. FASEB J. 2009;23:1490-1502.
72. Ravi V, Patel S. Vascular sarcomas. Curr Oncol Rep. 2013;15:347-355

73. von Mehren M, Rankin C, Goldblum JR, et al. Phase 2 Southwest Oncology Group-directed intergroup trial (S0505) of sorafenib in advanced soft tissue sarcomas. Cancer. 2012;118:770-776.

74. Penel N, Ray-Coquard I, Bal-Mahieu C, et al. Low level of baseline circulating VEGF-A is associated with better outcome in patients with vascular sarcomas receiving sorafenib: an ancillary study from a phase II trial. Target Oncol. 2014;9:273-277.

75. Hurwitz HI, Dowlati A, Saini S, et al. Phase I trial of pazopanib in patients with advanced cancer. Clin Cancer Res. 2009;15:4220-4227.

76. van der Graaf WT, Blay JY, Chawla SP, et al. Pazopanib for metastatic soft-tissue sarcoma (PALETTE): a randomised, double-blind, placebocontrolled phase 3 trial. Lancet. 2012;279:1879-1886.

77. Verweij J, Sleijfer S. Pazopanib, a new therapy for metastatic soft tissue sarcoma. Expert Opin Pharmacother. 2013;14:929-935.

78. Ogata D, Yanagisawa H, Suzuki K, Oashi K, Yamazaki N, Tsuchida T. Pazopanib treatment slows progression and stabilizes disease in patients with taxane-resistant cutaneous angiosarcoma. Med Oncol. 2016;33:116

79. Rosen A, Thimon S, Ternant D, Machet MC, Paintaud G, Machet L. Partial response to bevacizumab of an extensive cutaneous angiosarcoma of the face. Br J Dermatol. 2010;163:225-227.

80. Agulnik M, Yarber JL, Okuno SH, et al. An open-label, multicenter, phase II study of bevacizumab for the treatment of angiosarcoma and epithelioid hemangioendotheliomas. Ann Oncol. 2013;24:257-263.

81. Koontz BF, Miles EF, Rubio MA, et al. Preoperative radiotherapy and bevacizumab for angiosarcoma of the head and neck: two case studies. Head Neck. 2008;30:262-6.

82. Fuller CK, Charlson JA, Dankle SK, Russell TJ. Dramatic improvement of inoperable angiosarcoma with combination paclitaxel and bevacizumab chemotherapy. J Am Acad Dermatol. 2010;63: e83-e84.

83. Dickson MA, D'Adamo DR, Keohan ML, et al. Phase II trial of gemcitabine and docetaxel with bevacizumab in soft tissue sarcoma. Sarcoma. 2015;2015:532478.

84. Maki RG, Jungbluth AA, Gnjatic S, et al. A pilot study of anti-CTLA4 antibody ipilimumab in patients with synovial sarcoma. Sarcoma. 2013;2013:168145.

85. Sindhu S, Gimber LH, Cranmer L, McBride A, Kraft AS. Angiosarcoma treated successfully with anti-PD-1 therapy: a case report. $J$ Immunother Cancer 2017;5:58.

86. Lamm W, Natter C, Schur S, et al. Distinctive outcome in patients with non-uterine and uterine leiomyosarcoma. BMC Cancer. 2014;14:981.

87. Guo X, Jo VY, Mills AM, et al. Clinically relevant molecular subtypes in leiomyosarcoma. Clin Cancer Res. 2015;21:3501-2511.

88. Hernando E, Charytonowicz E, Dudas ME, et al. The AKT-mTOR pathway plays a critical role in the development of leiomyosarcoma. Nat Med. 2007;13:748-753.

89. Shi G, Perle MA, Mittal K, et al. Let-7 repression leads to HMGA2 overexpression in uterine leiomyosarcoma. J Cell Mol Med. 2009; 13:3898-3905.

90. Hayashi T, Horiuchi A, Sano K, et al. Potential Role of LMP2 as tumorsuppressor defines new targets for uterine leiomyosarcoma therapy. Sci Rep. 2011;1:180.

91. Shan W, Akinfenwa PY, Savannah KB, et al. A small-molecule inhibitor targeting the mitotic spindle checkpoint impairs the growth of uterine leiomyosarcoma. Clin Cancer Res. 2012;18:3352-3365.

92. Setia A, Kanotra S, Aggarwal R, Bhavthankar DP. Epithelioid leiomyosarcoma of uterus. BMJ Case Rep. 2012.

93. Edris B, Weiskopf K, Volkmer AK, et al. Antibody therapy targeting the CD47 protein is effective in a model of aggressive metastatic leiomyosarcoma. Proc Natl Acad Sci U S A. 2012;109:6656-6661.

94. Hu J, Rao UN, Jasani S, Khanna V, Yaw K, Surti U. Loss of DNA copy number of $10 \mathrm{q}$ is associated with aggressive behavior of leiomyosarcoma: a comparative genomic hybridization study. Cancer Genet Cytogenet. 2005;161:20-27. 
95. Hu J, Khanna V, Jones M, Surti U. Genomic alterations in uterine leiomyosarcomas: potential markers for clinical diagnosis and prognosis. Genes Chromosomes Cancer. 2001;31:117-124.

96. Poncelet C, Walker F, Medelenat P, et al. Expression of CD44 standard and isoforms V3 and V6 in uterine smooth muscle tumors: a possible diagnostic tool for the diagnosis of leiomyosarcoma. Hum Pathol. 2009;32:1190-1196.

97. Savannah KJ, Demicco EG. Dual targeting of mTOR and aurora-A kinase for the treatment of uterine leiomyosarcoma. Clin Cancer Res. 2012;18:4633-4645.

98. Schvartzman JM, Sotillo R, Benezra B. Mitotic chromosomal instability and cancer: mouse modeling of the human disease. Nat Rev Cancer. 2010;10:102-115.

99. Edris B, Espinosa I, Mühlenburg T, et al. ROR2 is a novel prognostic biomarker and a potential therapeutic target in leiomyosarcoma and gastrointestinal stromal tumour. J Pathol. 2012;227:223-233.

100. Giraudet AL, Badel JN, Cassier P, et al. SYNFRIZZ: a phase IA/IB of a radiolabelled monoclonal $\mathrm{AB}$ for the treatment of relapsing synovial sarcoma. J Nucl Med. 2014;55:223.

101. Sette G, Salvati V, Memeo L, et al. EGFR inhibition abrogates leiomyosarcoma cell chemoresistance through inactivation of survival pathways and impairment of CSC potential. PLoS One. 2012;7(10):e46891.

102. Hayashi T, Faustman DL. Development of spontaneous uterine tumors in low molecular mass polypeptide-2 knockout mice. Cancer Res. 2002;62:24-27.

103. Maki RG, Kraft AS, Scheu K, et al. A multicenter phase II study of bortezomib in recurrent or metastatic sarcomas. Cancer. 2005;103:1431-1438.

104. Gao CF, Xie Q, Zhang YW, et al. Therapeutic potential of hepatocyte growth factor/scatter factor neutralizing antibodies: inhibition of tumor growth in both autocrine and paracrine hepatocyte growth factor/scatter factor - c-Met-driven models of leiomyosarcoma. Mol Cancer Ther. 2009;8:2803-2810.

105. Burgess T, Coxon A, Meyer S, et al. Fully human monoclonal antibodies to hepatocyte growth factor with therapeutic potential against hepatocyte growth factor/c-Met-dependent human tumors. Cancer Res. 2006;66:1721-1729.

106. Iveson T, Donehower RC, Davidenko I, et al. Rilotumumab in combination with epirubicin, cisplatin, and capecitabine as first-line treatment for gastric or oesophagogastric junction adenocarcinoma: an open-label, dose de-escalation phase B study and a double-blind, randomized phase 2 study. Lancet Oncol. 2014;15:1007-1018.

107. Amgen. A multicenter, phase $1 / 1 \mathrm{~B}$, open label study evaluating the safety, tolerability and pharmacokinetics of rilotumumab in Japanese subjects. Available from: https://clinicaltrials.gov/ct2/show/NCT01791374. NLM identifier: NCT01791374. Accessed March 28, 2018.

108. Gynecologic Oncology Group. A phase II evaluation of AMG 102 (rilotumumab) (NSC \#750009) in the treatment of persistent or recurrent epithelial ovarian, fallopian tube, or primary peritoneal carcinoma. Available from: https:/clinicaltrials.gov/ct2/show/NCT01039207. NLM identifier: NCT01039207. Accessed March 28, 2018.

109. University of Pittsburgh. A phase I/II trial of AMG 102 and erlotinib in previously treated patients with advanced non-small cell lung cancer. Available from: https://clinicaltrials.gov/ct2/show/NCT01233687. NLM identifier: NCT01233687. Accessed March 28, 2018.

110. Duke University Medical Center. Phase II study to evaluate the efficacy and safety of AMG 102 and Avastin in subjects with recurrent malignant glioma. Available from: https:/clinicaltrials.gov/ct2/show/ NCT01113398. NLM identifier: NCT01113398. Accessed March 28, 2018.

111. Southwest Oncology Group. A biomarker-driven master protocol for previously treated squamous cell lung cancer (Lung-MAP). Available from: https:/clinicaltrials.gov/ct2/show/NCT02154490. NLM identifier: NCT02154490. Accessed March 28, 2018.

112. Paoluzzi L, Cacavio A, Ghesani M, et al. Response to anti-PD1 therapy with nivolumab in metastatic sarcomas. Clin Sarcoma Res. 2016;6:24.
113. Royal Marsden NHS Foundation Trust. A phase I study to assess the safety and tolerability of pembrolizumab in combination with fixed rate gemcitabine chemotherapy in patients with leiomyosarcoma and undifferentiated pleomorphic sarcoma. Available from: https://clinicaltrials.gov/ct2/show/NCT03123276. NLM identifier: NCT03123276. Accessed March 28, 2018.

114. Institut Bergonié. Combination of MK3475 and metronomic cyclophosphamide in patients with advanced sarcomas: multicentre phase II trial. Available from: https://clinicaltrials.gov/ct2/show/NCT02406781. NLM identifier: NCT02406781. Accessed March 28, 2018.

115. Heine A, Kristiansen G, Schild HH, Brossart P. Successful treatment of refractory leiomyosarcoma with PD-1 inhibitor nivolumab. Ann Oncol. 2016;27:1813-1814.

116. George S, Miao D, Demetri GD, et al. Loss of PTEN is associated with resistance to anti-PD-1 checkpoint blockade therapy in metastatic uterine leiomyosarcoma. Immunity. 2017;46:197-204.

117. Edris B, Weiskopf K, Weissman IL, van de Rijn M. Flipping the script on macrophages in leiomyosarcoma. Oncoimmunology. 2012;1: 1202-1204

118. Forty Seven. A phase I dose escalation trial of the humanized antiCD47 monoclonal antibody Hu5F9-G4 in haematological malignancies (CAMELLIA). Available from: https:/clinicaltrials.gov/ct2/show/ NCT02678338. NLM identifier: NCT02678338. Accessed March 28, 2018.

119. Celgene. A phase 1, open-label, dose finding study of CC-90002, a monoclonal antibody directed against CD47, in subjects with acute myeloid leukemia and high-risk myelodysplastic syndrome. Available from: https:/clinicaltrials.gov/ct2/show/NCT02641002. NLM identifier: NCT02641002. Accessed March 28, 2018.

120. Forty Seven. A first-in-human phase 1 dose escalation trial of Hu5F9G4 in patients with advanced solid malignancies. Available from: https://clinicaltrials.gov/ct2/show/NCT02216409. NLM identifier: NCT02216409. Accessed March 28, 2018.

121. Trillium Therapeutics. A phase $1 \mathrm{~A} / 1 \mathrm{~B}$ dose escalation and expansion trial of TTI-621, a novel biologic targeting CD47, in subjects with relapsed or refractory hematologic malignancies and selected solid tumors. Available from: https://clinicaltrials.gov/ct2/show/ NCT02663518. NLM identifier: NCT02663518. Accessed March 28, 2018.

122. Celgene. A phase I, open-label, dose finding study of CC-90002, a monoclonal antibody directed against CD47, in subjects with advanced solid and hematologic cancers. Available from: https://clinicaltrials.gov/ct2/show/NCT02367196. NLM identifier: NCT02367196. Accessed March 28, 2018.

123. Alexo Therapeutics. A phase 1, dose escalation study of ALX148 in patients with advanced solid tumors and lymphoma. Available from: https:/clinicaltrials.gov/ct2/show/NCT03013218. NLM identifier: NCT03013218. Accessed March 28, 2018.

124. Xia SJ, Pressey JG, Barr FG. Molecular pathogenesis of rhabdomyosarcoma. Cancer Biol Ther. 2002;1:97-104.

125. Huh WW, Skapek SX. Childhood rhabdomyosarcoma: new insight on biology and treatment. Curr Oncol Rep. 2010;12:402-410.

126. Ognjanovic S, Linabery AM, Charbonneau B, Ross JA. Trends in childhood rhabdomyosarcoma incidence and survival in the United States, 1975-2005. Cancer. 2009;115:4218-4226.

127. Hiniker SM, Donaldson SS. Recent advances in understanding and managing rhabdomyosarcoma. F1000Prime Rep. 2015;7:59.

128. Newton WA, Gehan EA, Webber BL, et al. Classification of rhabdomyosarcomas and related sarcomas: pathologic aspects and proposal for a new classification - an Intergroup Rhabdomyosarcoma Study. Cancer 1995;76:1073-1085

129. Terrier P, Henry-Amar M, Triche TJ, et al. Is neural-ectodermal differentiation of Ewing's sarcoma of bone associated with an unfavorable prognosis? Eur J Cancer. 1995;31A:307-314.

130. Hosoi H. Current status of treatment for pediatric rhabdomyosarcoma in the USA and Japan. Pediatr Int. 2016;58:81-87. 
131. Malempati S, Hawkins DS. Rhabdomyosarcoma: review of the Children's Oncology Group (COG) Soft-Tissue Sarcoma Committee experience and rationale for current COG studies. Pediatr Blood Cancer. 2012;59:5-10.

132. Shern JF, Chen L, Chmielecki J, et al. Comprehensive genomic analysis of rhabdomyosarcoma reveals a landscape of alterations affecting a common genetic axis in fusion-positive and fusion-negative tumors. Cancer Discov. 2014;4:216-231.

133. Huh WW, Skapek SX. Childhood rhabdomyosarcoma: new insight on biology and treatment. Curr Oncol Rep. 2010;12:402-410.

134. Sorensen PH, Lynch JC, Qualman SJ, et al. PAX3-FKHR and PAX7FKHR gene fusions are prognostic indicators in alveolar rhabdomyosarcoma: a report from the Children's Oncology Group. J Clin Oncol. 2002;20:2672-2679.

135. Fredericks WJ, Galili N, Mukhopadhyay S, et al. The PAX3-FKHR fusion protein created by the $\mathrm{t}(2 ; 13)$ translocation in alveolar rhabdomyosarcomas is a more potent transcriptional activator than PAX3. Mol Cell Biol. 1995; 15:1522-1535.

136. Anderson MJ, Shelton GD, Cavenee WK, Arden KC. Embryonic expression of the tumor-associated PAX3-FKHR fusion protein interferes with the developmental functions of Pax3. Proc Natl Acad Sci U S A. 2001;98:1589-1594.

137. Epstein JA, Song B, Lakkis M, Wang C. Tumor-specific PAX3-FKHR transcription factor, but not PAX3, activates the platelet-derived growth factor alpha receptor. Mol Cell Biol. 1998;18:4118-4130.

138. Lagutina I, Conway SJ, Sublett J, Grosveld GF. Pax3-FKHR knock-in mice show developmental aberrations but do not develop tumors. Mol Cell Biol. 2002;22:7204-7216.

139. Crose LE, Galindo KA, Kephart JG et al. Alveolar rhabdomyosarcomaassociated PAX3-FOXO1 promotes tumorigenesis via Hippo pathway suppression. J Clin Invest.2014;124:285-296.

140. Linardic CM, Naini S, Herndon JE 2nd, Kesserwan C, Qualman SJ, Counter CM. The PAX3-FKHR fusion gene of rhabdomyosarcoma cooperates with loss of p16INK4A to promote bypass of cellular senescence. Cancer Res. 2007;67:6691-6699.

141. Williamson D, Missiaglia E, de Reynies A, et al. Fusion genenegative alveolar rhabdomyosarcoma is clinically and molecularly indistinguishable from embryonal rhabdomyosarcoma. J Clin Oncol. 2010;28:2151-2158.

142. Barr FG, Duan F, Smith LM, et al. Genomic and clinical analyses of 2p24 and 12q13-14 amplification in alveolar rhabdomyosarcoma: a report from the Children's Oncology Group. Genes Chromosomes Cancer. 2009;48:661-672.

143. Loh WE Jr, Scrable HJ, Livanos E, et al. Human chromosome 11 contains two different growth suppressor genes for embryonal rhabdomyosarcoma. Proc Natl Acad Sci U S A. 1992;89:1755-1759.

144. Weber-Hall S, Anderson J, McManus A, et al. Gains, losses, and amplification of genomic material in rhabdomyosarcoma analyzed by comparative genomic hybridization. Cancer Res. 1996;56:3220-3224.

145. Bridge JA, Liu J, Weibolt V, et al. Novel genomic imbalances in embryonal rhabdomyosarcoma revealed by comparative genomic hybridization and fluorescence in situ hybridization: an intergroup rhabdomyosarcoma study. Genes Chromosomes Cancer. 2000;27: 337-344.

146. Gordon T, McManus A, Anderson J, et al. Cytogenetic abnormalities in 42 rhabdomyosarcoma: a United Kingdom Cancer Cytogenetics Group Study. Med Pediatr Oncol. 2001;36:259-267.

147. Stratton MR, Fisher C, Gusterson BA, Cooper CS. Detection of point mutations in N-ras and K-ras genes of human embryonal rhabdomyosarcomas using oligonucleotide probes and the polymerase chain reaction. Cancer Res. 1989;49:6324-6327.

148. Pressey JG, Anderson JR, Crossman DK, Lynch JC, Barr FG. Hedgehog pathway activity in pediatric embryonal rhabdomyosarcoma and undifferentiated sarcoma: a report from the Children's Oncology Group. Pediatr Blood Cancer. 2011;57:930-938.

149. Paulson V, Chandler G, Rakheja D, et al. High-resolution array CGH identifies common mechanisms that drive embryonal rhabdomyosarcoma pathogenesis. Genes Chromosomes Cancer. 2011;50:397-408.
150. Shukla N, Ameur N, Yilmaz I, et al. Oncogene mutation profiling of pediatric solid tumors reveals significant subsets of embryonal rhabdomyosarcoma and neuroblastoma with mutated genes in growth signaling pathways. Clin Cancer Res. 2012;18:748-757.

151. Takahashi Y, Oda Y, Kawaguchi K, et al. Altered expression and molecular abnormalities of cell-cycle-regulatory proteins in rhabdomyosarcoma. Mod Pathol. 2004;17:660-669.

152. Taylor AC, Shu L, Danks MK, et al. P53 mutation and MDM2 amplification frequency in pediatric rhabdomyosarcoma tumors and cell lines. Med Pediatr Oncol. 2000;35:96-103.

153. Hawkins DS, Gupta AA, Rudzinski ER. What is new in the biology and treatment of pediatric rhabdomyosarcoma? Curr Opin Pediatr. 2014;26:50-56.

154. Huang F, Greer A, Hurlburt W, et al. The mechanisms of differential sensitivity to an insulin-like growth factor-1 receptor inhibitor (BMS536924) and rationale for combining with EGFR/HER2 inhibitors. Cancer Res. 2009;69:161-170.

155. Renshaw J, Taylor KR, Bishop R, et al. Dual blockade of the PI3K/ AKT/mTOR (AZD8055) and RAS/MEK/ERK (AZD6244) pathways synergistically inhibits rhabdomyosarcoma cell growth in vitro and in vivo. Clin Cancer Res. 2013;19:5940-5951.

156. Guenther MK, Graab U, Fulda S. Synthetic lethal interaction between $\mathrm{PI} 3 \mathrm{~K} / \mathrm{Akt} / \mathrm{mTOR}$ and Ras/MEK/ERK pathway inhibition in rhabdomyosarcoma. Cancer Lett. 2013;337:200-209.

157. Chen X, Stewart E, Shelat AA, et al. Targeting oxidative stress in embryonal rhabdomyosarcoma. Cancer Cell. 2013;24:710-724.

158. Zhang M, Linardic CM, Kirsch DG. RAS and ROS in rhabdomyosarcoma. Cancer Cell. 2013;24:689-691.

159. Merchant MS, Bernstein D, Amoako M, et al. Adjuvant immunotherapy to improve outcome in high risk pediatric sarcomas. Clin Cancer Res. 2016;22:3182-3191.

160. National Cancer Institute. A pilot study of autologous T-cell transplantation with vaccine driven expansion of anti-tumor effectors after cytoreductive therapy in metastatic pediatric sarcomas. Available from: https://clinicaltrials.gov/ct2/show/NCT00001566. NLM identifier: NCT00001566. Accessed March 28, 2018.

161. National Cancer Institute. A pilot study of tumor vaccination and R-hIL-7 following standard multimodality therapy in patients with high risk pediatric solid tumors. Available from: https://clinicaltrials.gov/ ct2/show/NCT00923351. NLM identifier: NCT00923351. Accessed March 28, 2018.

162. Jennerex Biotherapeutics. A phase I, open-label, dose escalation study of JX-594 (vaccinia GM-CSF/thymidine kinase-deactivated virus) administered by intratumoral injection in pediatric patients with unresectable refractory solid tumors. Available from: https://clinicaltrials.gov/ct2/show/NCT01169584. NLM identifier: NCT01169584. Accessed March 28, 2018.

163. Nationwide Children's Hospital. A phase I dose escalation study of intratumoral or intravenous herpes simplex virus-1 mutant HSV1716 in patients with refractory non-central nervous system (non-CNS) solid tumors. Available from: https://clinicaltrials.gov/ct2/show/NCT00931931. NLM identifier: NCT00931931. Accessed March 28, 2018.

164. National Cancer Institute. A pilot study of tumor vaccination and R-hIL-7 following standard multimodality therapy in patients with high risk pediatric solid tumors. Available from: https://clinicaltrials.gov/ ct2/show/NCT00923351. NLM identifier: NCT00923351. Accessed March 28, 2018.

165. Baylor College of Medicine. Tumor associated antigen (TAA)-specific cytotoxic T-lymphocytes administered to patients with solid tumors. Available from: https://clinicaltrials.gov/ct2/show/NCT02239861. NLM identifier: NCT02239861. Accessed March 28, 2018.

166. Children's Oncology Group. A phase 2 study of IMGN901 (Lorvotuzumab Mertansine; NSC: 783609) in children with relapsed or refractory Wilms tumor, rhabdomyosarcoma, neuroblastoma, pleuropulmonary blastoma, malignant peripheral nerve sheath tumor (MPNST) and synovial sarcoma. Available from: https://clinicaltrials.gov/ct2/show/NCT02452554. NLM identifier: NCT02452554. Accessed March 28, 2018. 
167. De Alava E, Kawai A, Healey JH, et al. EWS-FLI1 fusion transcript structure is an independent determinant of prognosis in Ewing's sarcoma. J Clin Oncol. 1998;16:1248-1255.

168. Cotterill, SJ, Ahrens S. Prognostic factors in Ewing's tumor of bone: analysis of 975 patients from the European Intergroup Cooperative Ewing's Sarcoma Study group. J Clin Oncol. 2000;18(170): 3108-3114.

169. Angervall L, Enzinger FM. Extraskeletal neoplasm resembling Ewing's sarcoma. Cancer. 1975;36(1):240-251.

170. Sorensen PH, Lessnick SL, Lopez-Terrada D, Liu XF, Triche TJ, Denny CT. A second Ewing's sarcoma translocation, $t(21 ; 22)$, fuses the EWS gene to another ETS-family transcription factor, ERG. Nat Genet. 1994;6:146-151.

171. Siebenrock KA, Nascimento AG, Rock MG. Comparison of soft tissue Ewing's sarcoma and peripheral neuroectodermal tumor. Clin Orthop Relat Res. 1996:288-299.

172. Tanaka M, Yamazaki Y. Ewing's sarcoma precursors are highly enriched in embryonic osteochondrogenic progenitors. $J$ Clin Invest. 2014;124:3061-3074.

173. Riggi N, Stamenkovic I. The biology of Ewing sarcoma. Cancer Lett. 2007;254:1-10.

174. van Doominck JA, Ji L, Schaub B, et al. Current treatment protocols have eliminated the prognostic advantage of type 1 fusions in Ewing sarcoma: a report from the Children's Oncology Group. J Clin Oncol. 2010;28:1989-1994.

175. Crompton BD, Stewart C, Taylor-Weiner A, et al. The genomic landscape of pediatric Ewing sarcoma. Cancer Discov. 2014;4: 1326-1341.

176. 176. Tirode F, Surdez D, Ma X, et al. Genomic landscape of Ewing sarcoma defines an aggressive subtype with co-association of STAG2 and TP53 mutations. Cancer Discov. 2014;4:1342-1353.

177. May WA, Lessnick SL, Braun BS, et al. The Ewing's sarcoma EWS/ FLI-1 fusion gene encodes a more potent transcriptional activator and is a more powerful transforming gene than FLI-1. Mol Cell Biol. 1993;13:7393-7398.

178. Johnson KM, Mahler NR, Saund RS, et al. Role for the EWS domain of EWS/FLI in binding GGAA-microsatellites required for Ewing sarcoma anchorage independent growth. Proc Natl Acad Sci U S A. 2017;114:9870-9875

179. Kinsey M, Smith R, Lessnick SL. NR0B1 is required for the oncogenic phenotype mediated by EWS/FLI in Ewing's sarcoma. Mol Cancer Res. 2006;4:851-859.

180. Lu Q, Zhang Y, Ma L, et al. EWS-FLI1 positively regulates autophagy by increasing ATG4B expression in Ewing sarcoma cells. Int $J$ Mol Med. 2017;40:1217-1225.

181. He T, Surdex D, Rantala JK, et al. High-throughput RNAi screen in Ewing sarcoma cells identifies leucine rich repeats and WD repeat domain containing 1 (LRWD1) as a regulator of EWS-FLI1 driven cell viability. Gene. 2017;596:137-146.

182. Tanner JM, Bensard C, Wei P, et al. EWS/FLI is a master regulator of metabolic reprogramming in Ewing sarcoma. Mol Cancer Res. 2017;15:1517-1530.

183. Choo S, Wang P, Newbury R, Roberts W, Yang J. Reactivation of TWIST1 contributes to Ewing sarcoma metastasis. Pediatric Blood Cancer. 2018;65:e26721.

184. Sechler M, Parrish JK, Birks DK, Jedlicka P. The histone demethylase $\mathrm{KDM} 3 \mathrm{~A}$, and its downstream target MCAM, promote Ewing sarcoma cell migration and metastasis. Oncogene. 2017;36:4150-4160.

185. Peters HL, Yan Y, Nordgren TM, Cutucache CE, Joshi SS, Solheim JC. Amyloid precursor like protein 2 suppresses irradiation induced apoptosis in Ewing sarcoma cells and is elevated in immune evasive Ewing sarcoma cells. Cancer Biol Ther. 2013;14:752-760.

186. Subbiah V, Anderson P, Lazar AJ, Burdett E, Raymond K, Ludwig JA. Ewing's sarcoma: standard and experimental treatment options. Curr Treat Options Oncol. 2009;10:126-140.

187. Kovar H. Blocking the road, stopping the engine or killing the driver? Advances in targeting EWS/FLI-1 fusion in Ewing sarcoma as novel therapy. Expert Opin Ther Targets. 2014;18:1315-1328.
188. Aksnes LH, Bauer HC, Dahl AA, et al. Health status at long-term follow-up in patients treated for extremity localized Ewing sarcoma or osteosarcoma: a Scandinavian Sarcoma Group study. Pediatr Blood Cancer. 2009;53:84-89.

189. Parks R, Rasch EK, Mansky PJ, Oakley F. Differences in activities of daily living performance between long-term pediatric sarcoma survivors and a matched comparison group on standardized testing. Pediatr Blood Cancer. 2009;53:622-628.

190. Oncternal Therapeutics. TK216 in patients with relapsed or refractory Ewing sarcoma. Available from: https://clinicaltrials.gov/ct2/show/ NCT02657005. NLM identifier: NCT02657005. Accessed March 28, 2018.

191. Zöllner SK, Selvanathan SP, Graham GT. Inhibition of the oncogenic fusion protein EWS-FLI1 causes G2-M cell cycle arrest and enhanced vincristine sensitivity in Ewing's sarcoma. Sci Signal. 2017;10:eaam8429.

192. Spurny C, Kailayangiri S, Jamitzky S, et al. Programmed cell death ligand 1 (PD-L1) expression is not a predominant feature in Ewing sarcomas. Pediatr Blood Cancer. 2018;65:e26719.

193. Grohar PJ, Griffin LB, Yeung C, et al. Ecteinascidin 743 interferes with the activity of EWS-FLI1 in Ewing sarcoma cells. Neoplasia. 2011;13:145-153.

194. Baruchel S, Pappo A, Krailo M, et al. A phase 2 trial of trabectedin in children with recurrent rhabdomyosarcoma, Ewing sarcoma and nonrhabdomyosarcoma soft tissue sarcomas: a report from the Children's Oncology Group. Eur J Cancer. 2012;48:579-585.

195. Grohar PJ, Segars LE, Yeung C, et al. Dual targeting of EWS-FLI1 activity and the associated DNA damage response with trabectedin and SN38 synergistically inhibits Ewing sarcoma cell growth. Clin Cancer Res. 2014;20:1190-1203.

196. Erkizan HV, Kong Y, Merchant M, et al. A small molecule blocking oncogenic protein EWS-FLI1 interaction with RNA helicase A inhibits growth of Ewing's sarcoma. Nat Med. 2009;15:750-756.

197. Prieur A, Tirode F, Cohen P, Delattre O. EWS/FLI-1 silencing and gene profiling of Ewing cells reveal downstream oncogenic pathways and a crucial role for repression of insulin-like growth factor binding protein 3. Mol Cell Biol. 2004;24:7275-7283.

198. Malempati S, Weigel B, Ingle AM, et al. Phase I/II trial and pharmacokinetic study of cixutumumab in pediatric patients with refractory solid tumors and Ewing sarcoma: a report from the Children's Oncology Group. J Clin Oncol. 2012;30:256-262.

199. Pappo AS, Patel SR, Crowley J, et al. R1507, a monoclonal antibody to the insulin-like growth factor 1 receptor, in patients with recurrent or refractory Ewing sarcoma family of tumors: results of a phase II Sarcoma Alliance for Research Through Collaboration study. J Clin Oncol. 2011;29:4541-4547.

200. Lamhamedi-Cherradi SE, Menegaz BA, Ramamoorthy V. IGF-1R and mTOR blockade: novel resistance mechanisms and synergistic drug combinations for Ewing sarcoma. J Natl Cancer Inst. 2016;108:djw182.

201. Naing A, LoRusso P, Fu S, et al. Insulin growth factor-receptor (IGF1R) antibody cixutumumab combined with the mTOR inhibitor temsirolimus in patients with refractory Ewing's sarcoma family tumors. Clin Cancer Res. 2012;18:2625-2631.

202. Garnett MJ, Edelman EJ, Heidorn SJ, et al. Systematic identification of genomic markers of drug sensitivity in cancer cells. Nature. 2012;483:570-575.

203. Heske CM, Davis MI, Baumgart JT, et al. Matrix Screen identifies synergistic combination of PARP inhibitors and nicotinamide phosphoribosyltransferase (NAMPT) inhibitors in Ewing sarcoma. Clin Cancer Res. 2017;23:7301-7311.

204. Choy E, Butrynski JE, Harmon DC, et al. Phase II study of olaparib in patients with refractory Ewing sarcoma following failure of standard chemotherapy. BMC Cancer. 2014;14:813.

205. National Cancer Institute. NCI-COG pediatric MATCH (molecular analysis for therapy choice): a phase 2 subprotocol of olaparib in patients with tumors harboring defects in DNA damage repair genes. Available from: https://clinicaltrials.gov/ct2/show/NCT03233204. NLM identifier: NCT03233204. Accessed March 28, 2018. 
206. Massachusetts General Hospital. Phase I study of olaparib and temozolomide in adult patients with recurrent/metastatic Ewing's sarcoma following failure of prior chemotherapy. Available from: https://clinicaltrials.gov/ct2/show/NCT01858168. NLM identifier: NCT01858168. Accessed March 28, 2018.

207. Raj S, Bui M, Gonzales R, Letson D, Antonia SJ. Impact of PDL1 expression on clinical outcomes in subtypes of sarcoma. Ann Oncol. 2014;25:iv494-iv510.

208. Le DT, Uram JN, Wang H, et al. PD-1 blockade in tumors with mismatch-repair deficiency. $N$ Engl J Med. 2015;372:2509-2520.

209. Assaf-Harofeh Medical Center. A phase II single arm study assessing efficacy and safety of nivolumab plus ipilimumab in nonresectable/ metastatic sarcoma and endometrial carcinoma patients with somatic deficient MMR as a selection tool. Available from: https://clinicaltrials.gov/ct2/show/NCT02982486. NLM identifier: NCT02982486. Accessed March 28, 2018.

210. von Heyking K, Calzada-Wack J, Gollner S, et al. The endochondral bone protein CHM1 sustains an undifferentiated, invasive phenotype, promoting lung metastasis in Ewing sarcoma. Mol Oncol. 2017;11:1288-1301.

211. Thiel U, Pirson S, Müller-Spahn C, et al. Specific recognition and inhibition of Ewing tumor growth by antigen-specific allo-restricted cytotoxic T cells. Br J Cancer. 2011;104:948-956.

212. Blaeschke F, Thiel U, Kirschner A, et al. Human HLA-A 02-01/ CHM1+ allo-restricted $\mathrm{T}$ cell receptor transgenic CD8+ T cells specifically inhibit Ewing sarcoma growth in vitro and in vivo. Oncotarget. 2016;7:43267-43280.

213. Ghisoli M, Barve M, Schneider R, et al. Pilot Trial of FANG immunotherapy in Ewing's sarcoma. Mol Ther. 2015;23:1103-1109.

214. Gradalis. A two-part phase IIB trial of Vigil (Bi-shRNA-furin and GMCSF augmented autologous tumor cell immunotherapy) in Ewing's sarcoma. Available from: https://clinicaltrials.gov/ct2/show/ NCT02511132. NLM identifier: NCT02511132. Accessed March 28, 2018.

215. National Cancer Institute. A pilot study of tumor vaccination and R-hIL-7 following standard multimodality therapy in patients with high risk pediatric solid tumors. Available from: https://clinicaltrials.gov/ ct2/show/NCT00923351. NLM identifier: NCT00923351. Accessed March 28, 2018.

216. Spurrell EL, Fisher C, Thomas JM, Judson IR. Prognostic factors in advanced synovial sarcoma: an analysis of 104 patients treated at the Royal Marsden Hospital. Ann Oncol. 2005;16:437-444.

217. Nielsen TO, Poulin NM, Ladanyi M. Synovial sarcoma: recent discoveries as a roadmap to new avenues for therapy. Cancer Discov.2015;5:124-134.

218. Herzog CE. Overview of sarcomas in the adolescent and young adult population. J Pediatr Hematol Oncol. 2005;27:215-8.

219. Ladanyi M, Antonescu CR, Leung DH, et al. Impact of SYT-SSX fusion type on the clinical behavior of synovial sarcoma: a multi-institutional retrospective study of 243 patients. Cancer Res. 2002;62:135-140.

220. Sultan I, Rodriguez-Galindo C, Saab R, Yasir S, Casanova M, Ferrari A. Comparing children and adults with synovial sarcoma in the Surveillance, Epidemiology, and End Results program, 1983 to 2005:an analysis of 1268 patients. Cancer. 2009;115:3537-3547.

221. Fisher C. Synovial sarcoma. Ann Diagn Pathol. 1998;2:401-421.

222. Clark J, Rocques PJ, Crew AJ, et al. Identification of novel genes, SYT and SSX, involved in the $\mathrm{t}(\mathrm{X} ; 18)(\mathrm{p} 11.2 ; \mathrm{q} 11.2)$ translocation found in human synovial sarcoma. Nat Genet. 1994;7:502-508.

223. Nagayama S, Katagiri T, Tsunoda T, et al. Genome-wide analysis of gene expression in synovial sarcomas using a cDNA microarray. Cancer Res. 2002;62:5859-5866.

224. Weiss SW, Sobin LH. Histological Typing of Soft Tissue Tumours. 2nd ed. Heidelberg: Springer; 1994

225. Clark J, Rocques PJ, Crew AJ, et al. Identification of novel genes, SYT and SSX, involved in the $\mathrm{t}(\mathrm{X} ; 18)(\mathrm{p} 11.2 ; \mathrm{q} 11.2)$ translocation found in human synovial sarcoma. Nat Genet. 1994;7:502-508.
226. Skytting B, Nilsson G, Brodin B, et al. A novel fusion gene, SYT-SSX4, in synovial sarcoma. $J$ Natl Cancer Inst. 1999;91:974-975.

227. Kawai A, Woodruff J, Healey JH, Brennan MF, Antonescu CR, Ladanyi M. SYT-SSX gene fusion as a determinant of morphology and prognosis in synovial sarcoma. $N$ Engl J Med. 1998;338:153-160.

228. Antonescu CR, Kawai A, Leung DH, et al. Strong association of SYTSSX fusion type and morphologic epithelial differentiation in synovial sarcoma. Diagn Mol Pathol. 2000;9:1-8.

229. Nakaqawa Y, Numoto K, Yoshida A, et al. Chromosomal and genetic imbalances in synovial sarcoma detected by conventional and microarray comparative genomic hybridization. $J$ Cancer Res Clin Oncol. 2006;132:444-450

230. Szymanska J, Serra M, Skytting B, et al. Genetic imbalances in 67 synovial sarcomas evaluated by comparative genomic hybridization. Genes Chromosomes Cancer. 1998;23:213-219.

231. Allander SV, Illei PB, Chen Y, et al. Expression profiling synovial sarcoma by cDNA microarrays: association of ERBB2, IGFBP2, and ELF3 with epithelial differentiation. Am J Pathol. 2002;161:1587-1595.

232. Saito T, Oda Y, Sakamoto A, et al. Prognostic value of the preserved expression of the E-cadherin and catenin families of adhesion molecules and of B-catenin mutations in synovial sarcoma. $J$ Pathol. 2000;192:342-350.

233. Nielsen T, Hsu FD, O'Connell J, et al. Tissue microarray validation of epidermal growth factor receptor and SALL2 in synovial sarcoma with comparison to tumors of similar histology. Am J Pathol. 2003:163:1449-1456.

234. Terry J, Saito T, Subramanian S, et al. TLE1 as a diagnostic immunohistochemical marker for synovial sarcoma emerging from gene expression profiling studies. Am J Surg Pathol. 2007;31:240-246.

235. Washington University School of Medicine. A phase I/II clinical trial of dose-escalating DHEA in synovial sarcoma patients. Available from: https://clinicaltrials.gov/ct2/show/NCT02683148. NLM identifier: NCT02683148. Accessed March 28, 2018.

236. Epizyme. A phase 1 study of the EZH2 inhibitor tazemetostat in pediatric subjects with relapsed or refractory INI1-negative tumors or synovial sarcoma. Available from: https://clinicaltrials.gov/ct2/show/ NCT02601937. NLM identifier: NCT02601937. Accessed March 28, 2018.

237. Epizyme. A phase II, multicenter study of the EZH2 inhibitor tazemetostat in adult subjects with INI1-negative tumors or relapsed/refractory synovial sarcoma. Available from: https://clinicaltrials.gov/ct2/show/ NCT02601950. NLM identifier: NCT02601950. Accessed March 28, 2018.

238. Advenchen Laboratories. A phase III study of AL3818 (anlotinib) hydrochloride monotherapy in subjects with metastatic or advanced alveolar soft part sarcoma, leiomyosarcoma, and synovial sarcoma. Available from: https://clinicaltrials.gov/ct2/show/NCT03016819. NLM identifier: NCT03016819. Accessed March 28, 2018.

239. National Cancer Institute. A phase $1 B / 2$ study of imatinib in combination with everolimus in synovial sarcoma. Available from: https://clinicaltrials.gov/ct2/show/NCT01281865. NLM identifier: NCT01281865. Accessed March 28, 2018.

240. Memorial Sloan Kettering Cancer Center. Phase II trial of sorafenib and dacarbazine in soft tissue sarcoma. Available from: https://clinicaltrials.gov/ct2/show/NCT00837148. NLM identifier: NCT00837148. Accessed March 28, 2018.

241. Kawaguchi S, Wada T, Ida K, et al. Phase I vaccination trial of SYTSSX junction peptide in patients with disseminated synovial sarcoma. J Transl Med. 2005;3:1.

242. Suminoe A, Matsuzaki A, Hattori H, Koga Y, Hara T. Immunotherapy with autologous dendritic cells and tumor antigens for children with refractory malignant solid tumors. Pediatr Transplant. 2009; 13:746-753.

243. Kawaguchi S, Tsukahara T, Ida K, et al. SYT-SSX breakpoint peptide vaccines in patients with synovial sarcoma: a study from the Japanese Musculoskeletal Oncology Group. Cancer Sci. 2012;103:1625-1630. 
244. Jungbluth AA, Antonescu CR, Busam KJ, et al. Monophasic and biphasic synovial sarcomas abundantly express cancer/testis antigen NY-ESO-1 but not MAGE-A1 or CT7. Int J Cancer. 2001;94: 252-256.

245. Robbins PF, Morgan RA, Feldman SA, et al. Tumor regression in patients with metastatic synovial cell sarcoma and melanoma using genetically engineered lymphocytes reactive with NY-ESO-1. J Clin Oncol. 2011;29:911-924.

246. Jager E, Nagata Y, Gnjatic S, et al. Monitoring CD8 T cell responses to NY-ESO-1: correlation of humoral and cellular immune responses. Proc Natl Acad Sci U S A. 2000;97:4760-4765.

247. Stockert E, Jager E, Chen YT, et al. A survey of the humoral immune response of cancer patients to a panel of human tumor antigens. $J \operatorname{Exp}$ Med. 1998;187:1349-1354.

248. van den Eynde BJ, van der Bruggen P. T cell defined tumor antigens. Curr Opin Immunol. 1997;9:684-693.

249. Adaptimmune. A pilot study of genetically engineered NY-ESO-1 specific NY-ESO-1 C259T in HLA-A2+ patients with synovial sarcoma. Available from: https:/clinicaltrials.gov/ct2/show/NCT01343043. NLM identifier: NCT01343043. Accessed March 28, 2018.

250. National Cancer Institute. Phase II study of metastatic cancer that expresses NY-ESO-1 using lymphodepleting conditioning followed by infusion of anti-NY-ESO-1 murine TCR-gene engineered lymphocytes. Available from: https://clinicaltrials.gov/ct2/show/ NCT01967823. NLM identifier: NCT01967823. Accessed March $28,2018$.
251. Takara Bio. Multi-center phase I/II study of NY-ESO-1 T cell receptor gene transferred $\mathrm{T}$ lymphocytes in patients with synovial sarcoma. Available from: https://clinicaltrials.gov/ct2/show/NCT03250325. NLM identifier: NCT03250325. Accessed March 28, 2018.

252. Immune Design. A randomized, open-label, phase II trial of CMB305 (sequentially administered LV305 and G305) and atezolizumab in patients with locally advanced, relapsed, or metastatic sarcoma expressing NYESO-1. Available from: https://clinicaltrials.gov/ct2/show/NCT02609984. NLM identifier: NCT02609984. Accessed March 28, 2018.

253. Petrov Research Institute of Oncology. Non-randomized single-center study phase II evaluating the efficacy and toxicity of autologous dendritic cell vaccine loaded with allogenic tumor lysate expression of cancer testis antigens in patients with soft tissue sarcoma. Available from: https://clinicaltrials.gov/ct2/show/NCT01883518. NLM identifier: NCT01883518. Accessed March 28, 2018.

254. Zhang P, Pollock RE. Epigenetic regulators: new therapeutic targets for soft tissue sarcoma. Cancer Cell Microenviron. 2014;1:e191.

255. Kawaguchi K, Oda Y, Saito T, et al. DNA hypermethylation status of multiple genes in soft tissue sarcomas. Mod Pathol. 2006;14:106-114.

256. Lubieniecka JM, de Bruijn DR, Su L, et al. Histone deacetylase inhibitors reverse SS18-SSX-mediated polycomb silencing of the tumor suppressor early growth response 1 in synovial sarcoma. Cancer Res. 2008;68:4303-4310.

257. Wang H, Garzon R, Sun H, et al. NF-кB-YY1-miR-29 regulatory circuitry in skeletal myogenesis and rhabdomyosarcoma. Cancer Cell. 2008;14:369-381.
Cancer Management and Research

\section{Publish your work in this journal}

Cancer Management and Research is an international, peer-reviewed open access journal focusing on cancer research and the optimal use of preventative and integrated treatment interventions to achieve improved outcomes, enhanced survival and quality of life for the cancer patient. The manuscript management system is completely online and includes

\section{Dovepress}

a very quick and fair peer-review system, which is all easy to use. Visit http://www.dovepress.com/testimonials.php to read real quotes from published authors. 